\title{
A Shallow Water Spectral Wave Model
}

\author{
IAN R.YOUNG
}

\author{
Department of Civl Engineering, Australian Defence Force Academy, Canberra
}

\begin{abstract}
A shallow water spectral wave prediction model based on a numerical solution of the radiative transfer equation is presented. The model is second generation and uses a simple yet effective representation for the nonlinear source term. In addition, the model pays particular attention to the shallow water processes of refraction, shoaling, bottom friction, and wave breaking. The flexibility of the model is demonstrated by an intercomparison with field data from a number of tropical cyclones. The turning winds from such storms provide a demanding test of model physics. The comparisons are in both deep and shallow water.
\end{abstract}

\section{INTRODUCTION}

Wave prediction models based on numerical solutions of the radiative transfer equation [Hasselmann, 1960] have made considerable progress since their inception. First generation models Gelci, et al., 1956; Pierson, et al., 1966; Inoue, 1967; Isozaki and $U_{j i,}$ 1973; Cardone, et al., 1975; Cavaleri and Rizzoli, 1981; Chen and Wang, 1983] considered the evolution of the deep water spectrum to be the result of the balance between atmospheric input and white cap dissipation. Hasselmann [1962] showed that the spectral balance was more complex than assumed in first generation models with nonlinear wave-wave interactions playing an important role. The inclusion of such terms in a spectral model poses considerable problems due to the computational expense required to calculate the nonlinear terms. Second generation models [Barnett, 1968; Ewing, 1971; Golding, 1983; Allender, et al., 1985; Greenwood, et al., 1985; Sobey and Young, 1987] overcame this problem by representing the nonlinear terms in a parametric form based on the spectral shape. The inclusion of the parametric representation generally improves the performance of such models as compared to first generation models. Due to the relatively small number of parameters used to represent the nonlinear term, however, second generation models have difficulty in representing the complex spectra generated by rapidly changing wind fields [ $S W A M P$ Group, 1985]. S. Hasselmann et al. [1985] and Komen [1987] have proposed a third generation model which uses a discrete interaction approximation to represent the nonlinear terms. This representation considers only a very small subset of the possible wave number components which can interact in a full solution. This approach has the advantage of retaining the physics of the interaction process. Consequently, performance in complex sea conditions should be enhanced.

The extension of any of these classes of model to water of finite depth requires the inclusion of the additional processes of refraction, shoaling, bottom dissipation, and depth limited breaking. The first attempts at such a model were made by Collins [1972] who developed a single-point, first generation model for finite water depth. Similar two-dimensional models have been developed by Cavaleri and Rizzoli [1981] and Chen and Wang [1983].

Copyright 1988 by the American Geophysical Union.

Paper number $8 \mathrm{C0064.}$

0148-0227/88/008C-0064\$05.00
This paper describes a second generation shallow water model. The model, called ADFA1, uses a simple yet very flexible representation for the nonlinear terms which overcomes many of the limitations of other second generation models. To demonstrate the applicability of the model, a very extensive intercomparison with field data under tropical cyclone conditions is presented. These data were obtained in both deep and shallow water. The rapidly turning winds of tropical cyclones provide a very demanding test of model physics.

\section{Radiative Transfer Equation}

The evolution of the directional wave spectrum $E(f, \theta$; $x, y, t)$ in water of finite depth can be described by the radiative transfer equation [Sobey, 1986]

$$
\begin{aligned}
& \frac{\partial}{\partial t}\left(C C_{g} E\right)+C_{g} \cos \theta \frac{\partial}{\partial x}\left(C C_{g} E\right)+C_{g} \sin \theta \frac{\partial}{\partial y}\left(C C_{g} E\right) \\
& +\frac{C_{g}}{C}\left[\sin \theta \frac{\partial C}{\partial x}-\cos \theta \frac{\partial C}{\partial y}\right] \frac{\partial}{\partial \theta}\left(C C_{g} E\right)=C C_{g} S
\end{aligned}
$$

where $C(x, y ; f)$ is the wave phase speed and $C_{g}(x, y ; f)$ is the wave group velocity. The terms on the left-hand side of (1) represent respectively local or temperal accumulation, propagation (terms 2 and 3 ) and combined refraction and shoaling (terms 4 and 5). The source term $S(f, \theta ; x, y, t)$ represents the net transfer of energy to, from or within the spectrum.

In addition, the kinematics of wave propagation are described by ray theory. In the absence of currents, frequency is constant along wave rays which are described by the characteristic equations [Munk and Arthur, 1952]

$$
\begin{gathered}
\frac{d x}{d t}=C_{g} \cos \theta \\
\frac{d y}{d t}=C_{g} \sin \theta \\
\frac{d \theta}{d t}=\frac{C_{g}}{C}\left[\sin \theta \frac{\partial C}{\partial x}-\cos \theta \frac{\partial C}{\partial y}\right]
\end{gathered}
$$

In deep water both $C$ and $C_{g}$ are independent of depth, the characteristics become straight lines, and (1) reduces to the deep-water form utilized by the models mentioned earlier.

Solution of (1) along the wave characteristics yields 


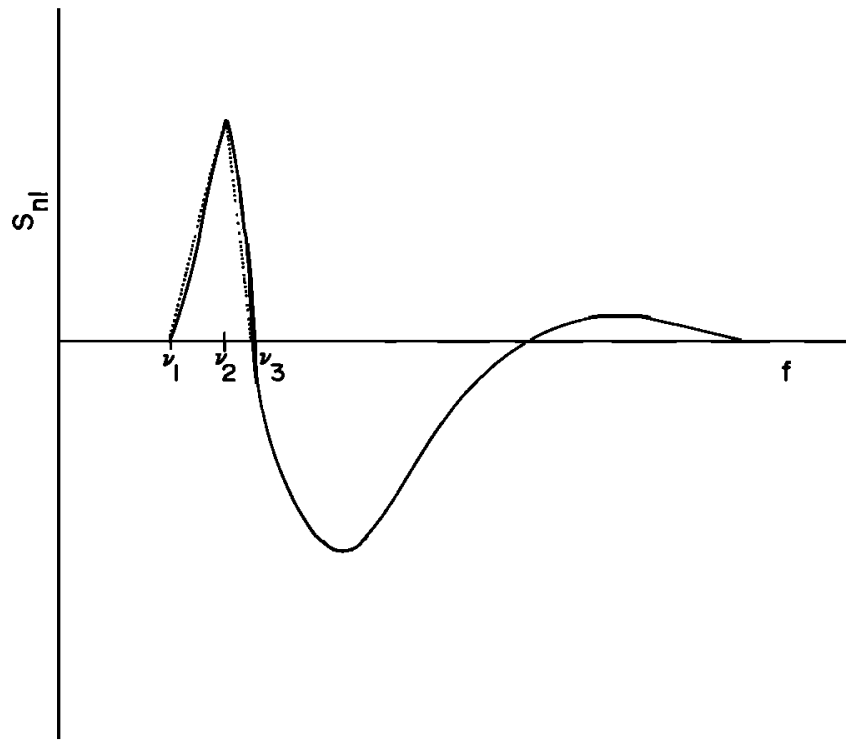

Fig. 1. Parametric representation of the forward lobe of the nonlinear source term.

$$
\frac{d}{d t}\left(C C_{g} E\right)=C C_{g} S
$$

In the absence of forcing, $S=0$ and (3) becomes

$$
\frac{d}{d t}\left(C C_{g} E\right)=0
$$

indicating that the quantity $C C_{g} E$ is conserved along the wave characteristics. In deep water, $C$ and $C_{g}$ are invarient, and $E$ is conserved along wave characteristics.

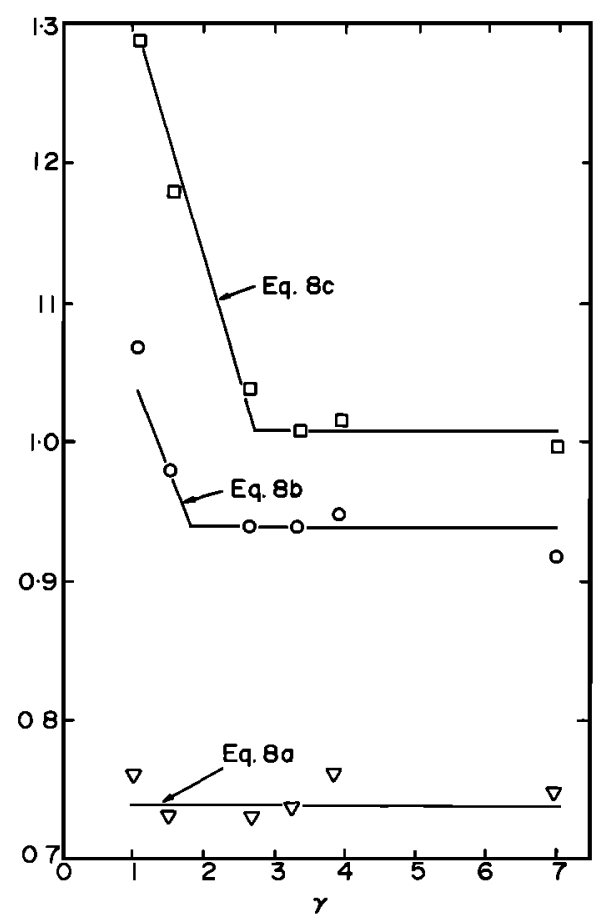

Fig. 2. Dependence of the nondimensional frequency factors $\nu_{1}, \nu_{2}$, and $\nu_{3}$ on the peak enhancement factor $\gamma$. The piecewise linear approximations, Equations (8) are shown together with the results of Hasselmann and Hasselmann [1981].
TABLE 1. Values of the Forward Lobe Peak of $S_{n l}$ for the Reference JONSWAP Spectrum

\begin{tabular}{cc}
$\begin{array}{c}\text { Angle from Mean } \\
\text { Spectral Direction }\end{array}$ & $\begin{array}{c}\text { Magnitude of Peak } \\
\text { Nonlinear Transfer } \\
\left(\mathrm{m}^{2}\right)\end{array}$ \\
\hline $0^{\circ}$ & $3.3 \times 10^{-5}$ \\
$15^{\circ}$ & $2.8 \times 10^{-5}$ \\
$30^{\circ}$ & $1.7 \times 10^{-5}$ \\
$45^{\circ}$ & $0.7 \times 10^{-5}$ \\
$60^{\circ}$ & 0.0 \\
\hline
\end{tabular}

The net source term $S$ is represented as the summation of a number of separate influences [Hasselmann et al., 1973]

$$
S=S_{\mathrm{in}}+S_{\mathrm{nl}}+S_{\mathrm{dis}}+S_{\mathrm{bf}}+S_{\mathrm{brk}}
$$

representing atmospheric input to the spectrum, nonlinear interactions within the spectrum, white cap dissipation, shallow water decay due to bottom friction, and depth-limited wave breaking, respectively.

\section{SOURCE TERMS}

Atmospheric Input, $S_{\text {in }}$

Atmospheric input is based on the field measurements of Snyder et al. [1981]

$$
S_{\text {in }}=b E(f, \theta)
$$

where

$b(f, \theta)= \begin{cases}0.25 \frac{\rho_{a}}{\rho_{w}}\left[\frac{U_{10}}{C} \cos \left(\theta-\theta_{w}\right)-1\right] \omega & \left|\theta-\theta_{w}\right|<90^{\circ} \\ 0 & \left|\theta-\theta_{w}\right| \geq 90^{\circ}\end{cases}$

where $\rho_{a}$ and $\rho_{w}$ are the densities of air and water, respectively, $\theta_{w}$ is the wind direction, $f$ the frequency, and $\omega=2 \pi f$. Komen et al. [1984] have suggested that (7) should be rescaled in terms of $u_{*}$ instead of $U_{10}$. Although such a representation would appear to have a sounder physical basis, lack of data on the appropriate drag coefficient to use, particularly at high wind speeds, leads to problems with its implementation in a wave model. More recently, Plant [1982] has suggested the scaling should be in terms of $\left(u_{*} / C\right)^{2}$, and Hsiao and Shemdin [1983] and D. Hasselmann, et al. [1985] have proposed $\left(U_{10} / C-1\right)^{2}$ rather than $\left(U_{10} / C-1\right)$. Although there is still debate, $(7)$ has been adopted for use in ADFAl. For waves moving slower or in opposition to the wind, (7) indicates there will be no decay. This is in agreement with the measurements of Young and Sobey [1985] and D. Hasselmann, et al. [1985], who indicate that such decay is quite small. As no linear or Phillips' mechanism [Phillips, 1957] is included in (6), initial growth from a zero energy state is achieved by using a "seed" spectrum. This spectrum has the standard JONSWAP form with parameters $\alpha=0.02, \gamma=2.26, \sigma_{a}=0.07, \sigma_{b}=0.09$, and $f_{m}$ equal to the second highest frequency bin specified 


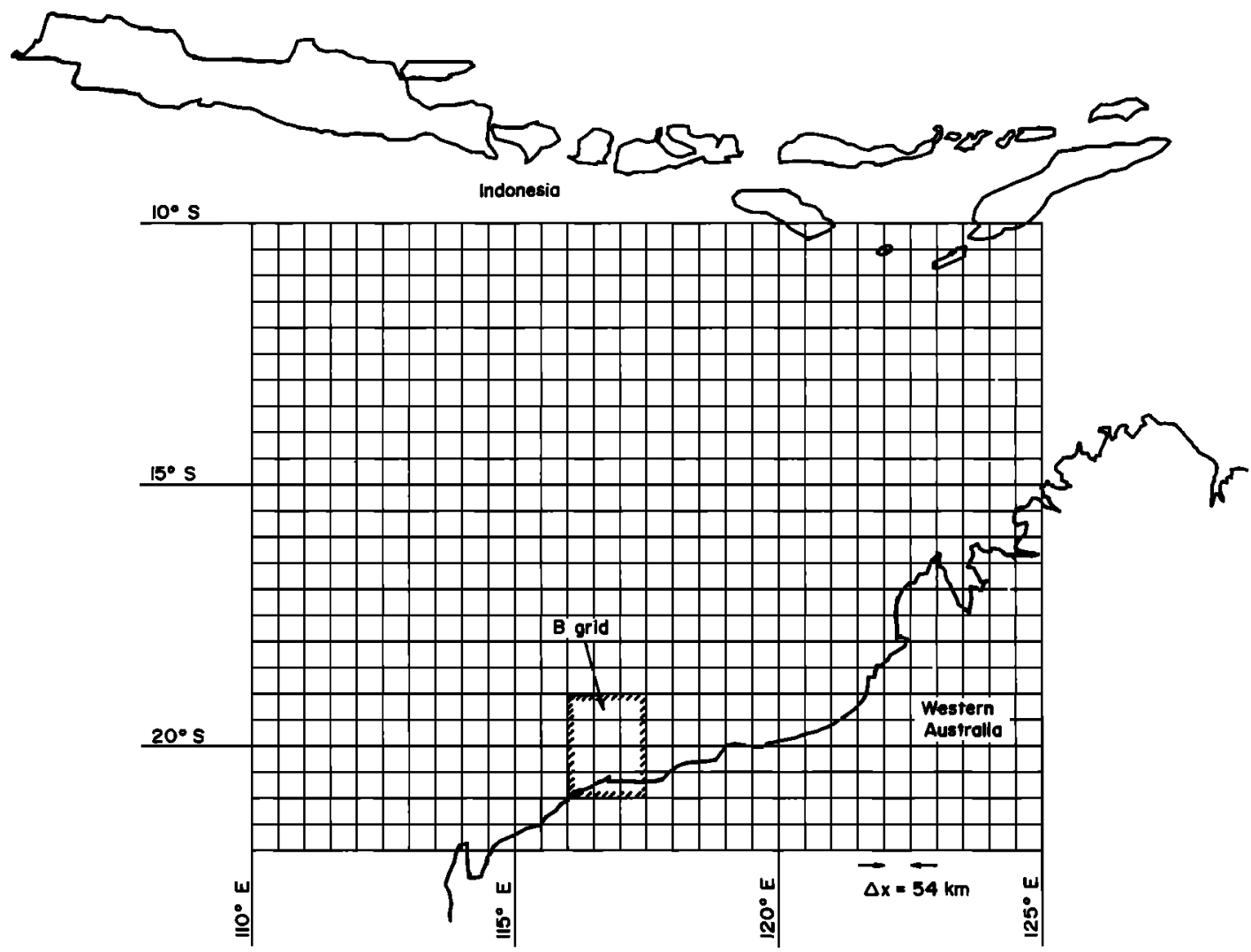

Fig. 3a. The A grid for the tropical cyclone simulation.

in the discrete model (typically $0.5 \mathrm{~Hz}$ ). The actual form of the spectrum is not important and is quickly modified by the source terms; it serves only to trigger growth in a similar fashion to the Phillips' mechanism.

\section{Nonlinear Interactions, $S_{\mathrm{nl}}$}

Although the nonlinear source term is of comparable magnitude to the other terms in the spectral balance, the numerical experiments of Komen et al. [1984] and Young et al. [1985] clearly illustrate the dominant role it plays in the evolution of the spectrum. Consequently, a flexible and accurate representation is required if reliable predictions are to be achieved under a wide variety of situations.

Hasselmann and Hasselmann [1981] have shown that for spectra which conform to the general JONSWAP shape, the nonlinear term can be scaled from results for the standard JONSWAP spectrum. Tests with this formulation, however, indicated that these scaling relationships did not position the positive lobe of the spectral transfer accurately enough to produce suitable results.

It is the magnitude and position of the forward lobe of the nonlinear transfer which plays a vital role in the evolution of the spectrum. The negative lobe has a far less significant role and accounts mainly for the "overshoot/undershoot" phenomenum [Young et al., 1987] as well as maintaining the spectral balance in the region to the right of the spectral peak. In a second generation model, however, this balance is maintained by the application of a spectral saturation level. Hence there seems little point in attempting to parameterize this section of the nonlinear transfer. Adopting such an approach enables a much more accurate representation of the forward lobe.

Following the general approach of Hasselmann and Hasselmann [1981], the forward lobe is scaled from the results of the mean JONSWAP spectrum. From the extensive calculations of Hasselmann and Hasselmann [1981] the position of the forward lobe is represented in terms of the nondimensional frequency, $\nu=f / f_{m}$, where $f_{m}$ is the frequency of the spectral peak. In view of the strongly peaked nature of the lobe and the discrete frequency representation required in spectral wave models, a triangular distribution was found to be a sufficiently accurate representation of the forward lobe. To define the position of the transfer in frequency space, it is only necessary to determine the values $\nu_{1}, \nu_{2}$, and $\nu_{3}$ shown in Figure 1.

Examination of the Hasselmann and Hasselmann [1981] results indicate that $\nu_{1}, \nu_{2}$, and $\nu_{3}$ depend only on the peak enhancement factor $\gamma$. A piecewise linear approximation to this data is shown in Figure 2 and can be represented by

$$
\begin{gathered}
\nu_{1}=0.74 \\
\nu_{2}=\max (0.94,1.17-0.13 \gamma) \\
\nu_{3}=\max (1.01,1.457-0.167 \gamma)
\end{gathered}
$$

The magnitude of the nonlinear transfer is a function of the JONSWAP parameters $\gamma, \alpha$ and $f_{m}$. The dependence on $\alpha$ and $f_{m}$ can be shown theoretically to be [Hasselmann and Hasselmann, 1981] 


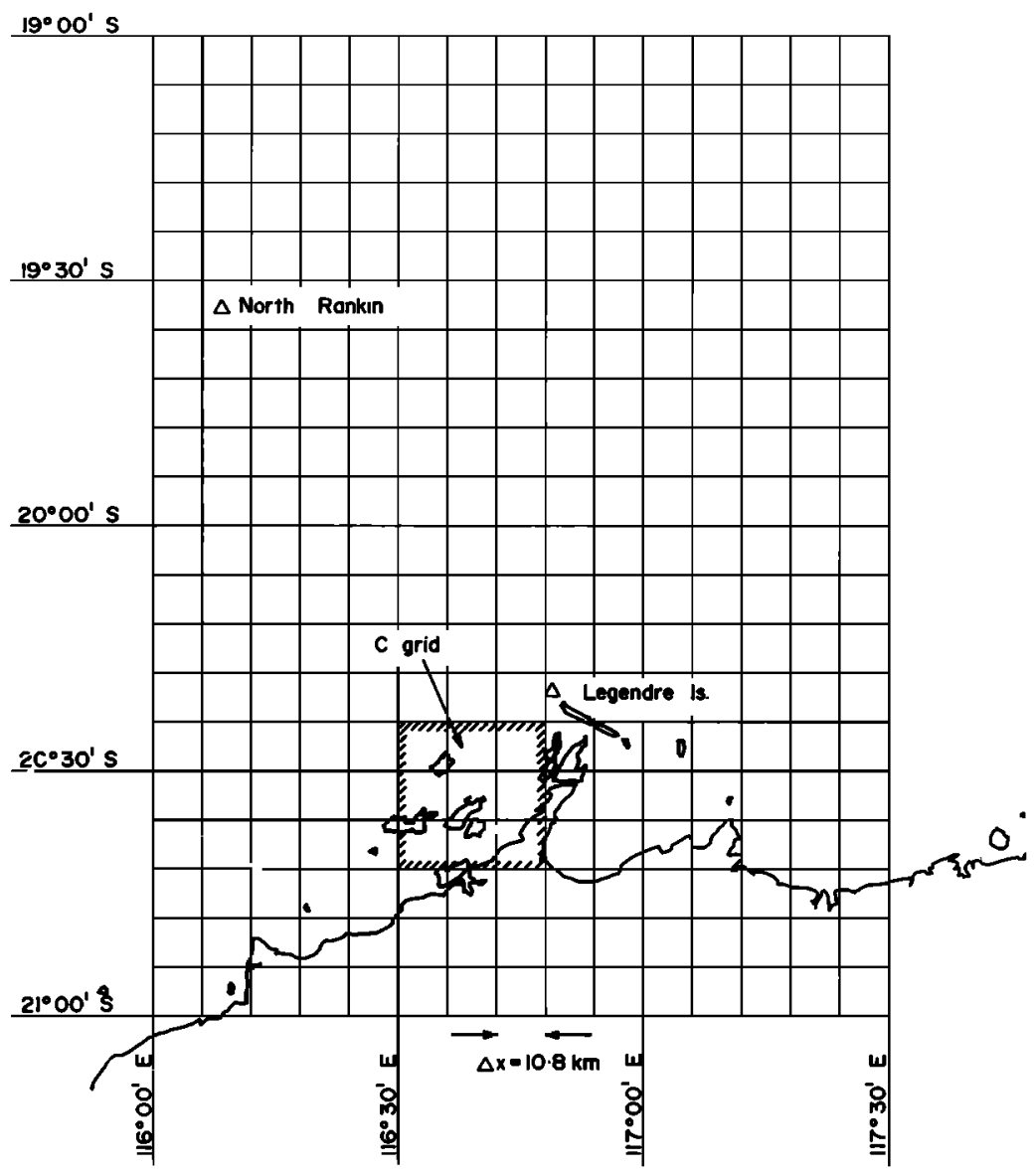

Fig. 3b. The B grid for the tropical cyclone simulation. The data recording sites at North Rankin and Legendre Island are shown as triangles.

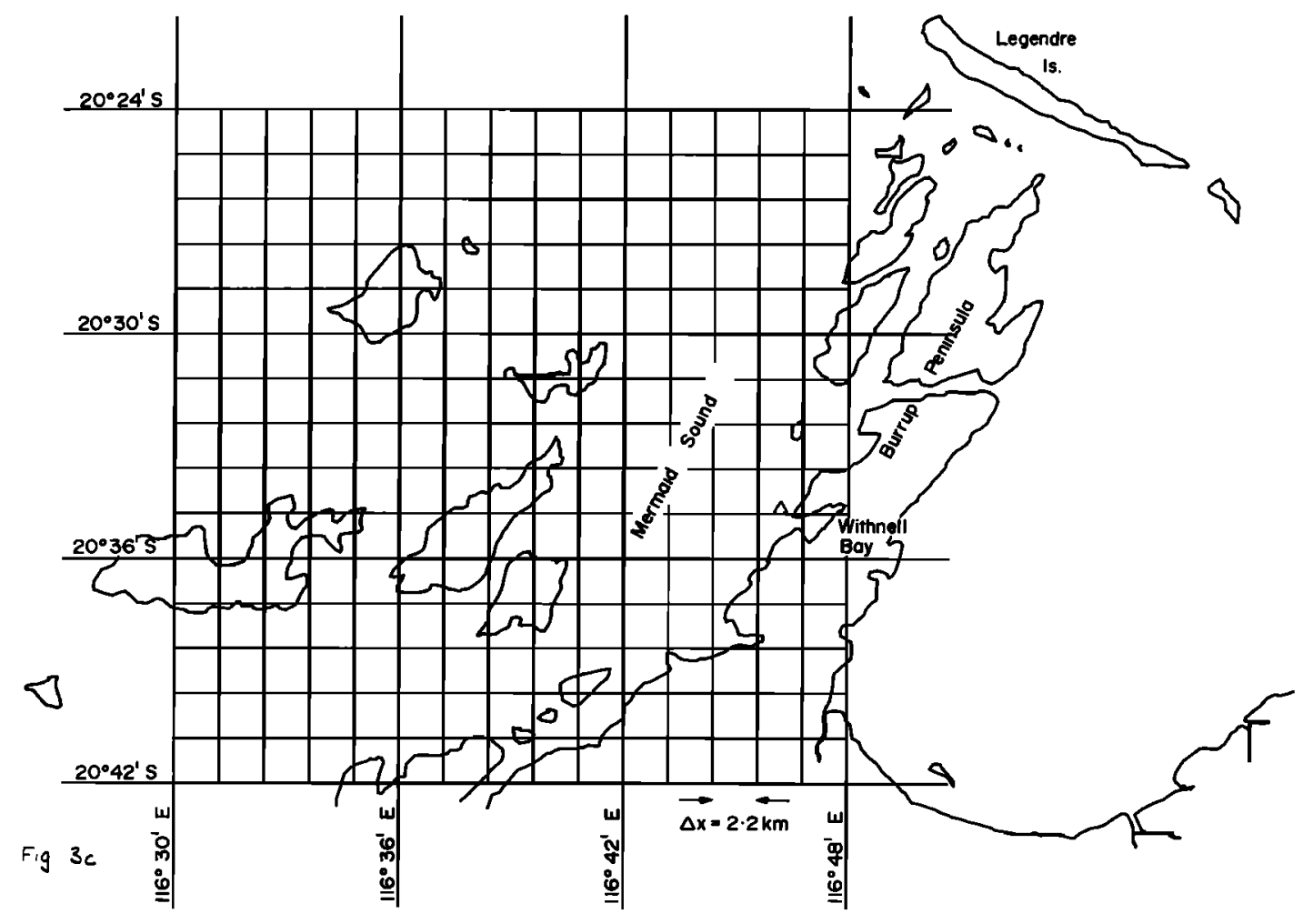

Fig. 3c. The $\mathrm{C}$ grid for the tropical cyclone simulation. The data recording site at Withnell Bay is shown as a triangle. 


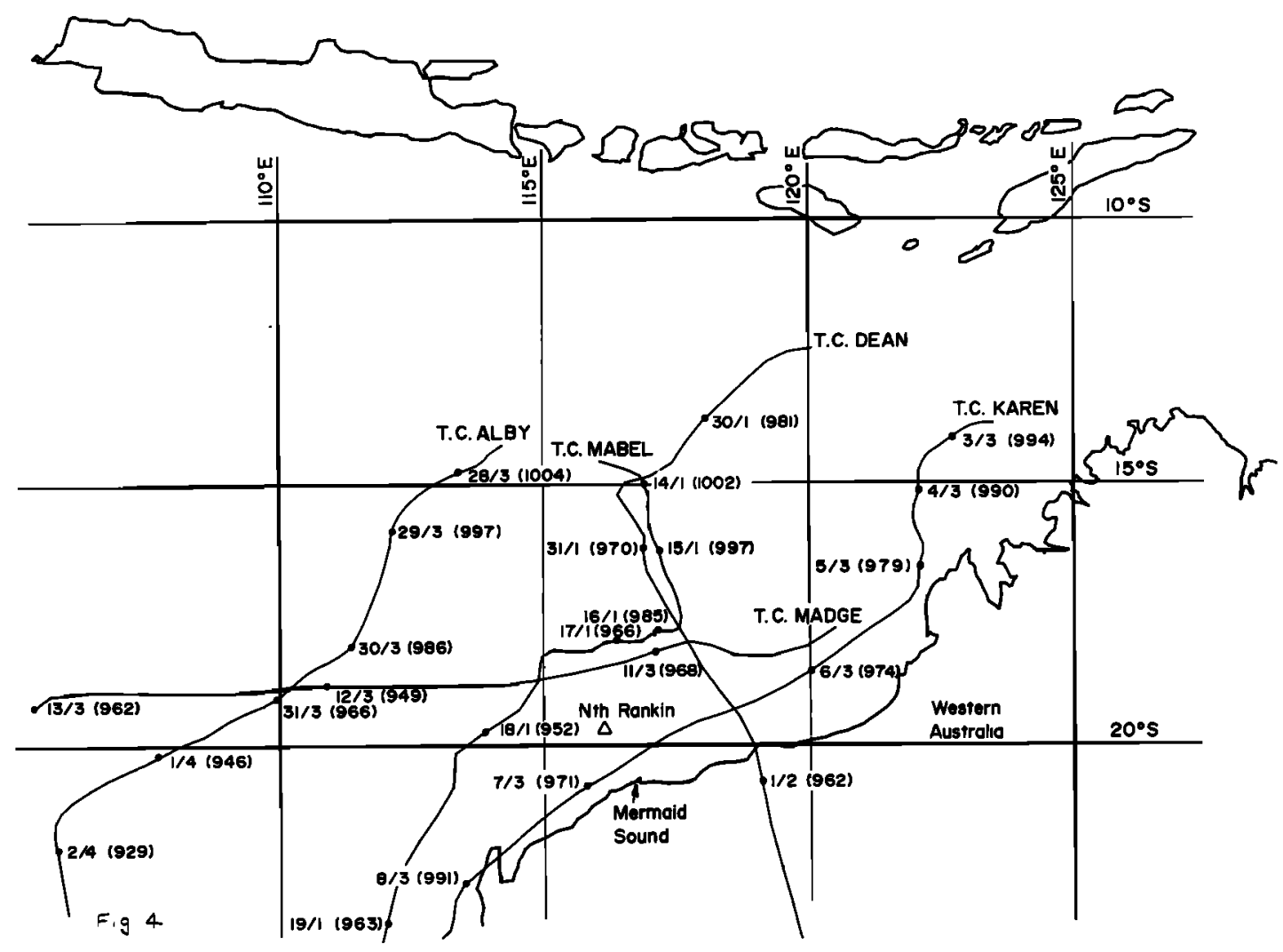

Fig. 4. Tropical cyclone storm tracks. The central positions of the storms are shown at 1200 with the day of the month followed by the month and the central pressure in brackets.

$$
S_{\mathrm{nl}}=S_{\mathrm{n} l}^{\prime}\left(\frac{\alpha}{\alpha^{\prime}}\right)^{3}\left(\frac{f_{m}}{f_{m}^{\prime}}\right)^{-4}
$$

where $S_{\mathrm{nl}}^{\prime}, \alpha^{\prime}$, and $f_{m}^{\prime}$ are the values associated with the reference JONSWAP spectrum from which the transfer is being scaled and $S_{\mathrm{nl}}, \alpha$, and $f_{m}$ are associated with the spectrum for which the nonlinear transfer is being calculated.

The dependence on $\gamma$ is less well defined, but the numerical experiments of Hasselmann and Hasselmann [1981] have provided a data set from which a scaling factor was developed. This $\gamma$ dependent scaling factor $A(\gamma)$ was represented as a polynomial approximation to the data:

$$
\begin{aligned}
A(\gamma)= & 1.0456 \times 10^{-2}+9.9260 \times 10^{-2} \gamma-1.5361 \times 10^{-2} \gamma^{2} \\
& +2.5330 \times 10^{-2} \gamma^{3}-1.2505 \times 10^{-3} \gamma^{4}
\end{aligned}
$$

Provided $\gamma, f_{m}$, and $\alpha$ are known for any spectrum, application of this parametric approach is quite straightforward. The mean JONSWAP spectrum with $\gamma=3.3$, $f_{m}=0.3$, and $\alpha=0.01$ is taken as the base data set for which all results are scaled. Since a triangular distribution for the forward lobe is assumed, it is only necessary to store the values for the peak of the positive lobe of the transfer associated with this reference spectrum. Values at angles of $0^{\circ}, 15^{\circ}, 30^{\circ}, 45^{\circ}$, and $60^{\circ}$ are used as shown in Table 1.

For a given angle to the mean spectral direction, the peak value is determined by linear interpolation within Table 1.
This value is then scaled according to the relationships given in (9) and (10). The scaled lobe is then positioned in frequency space according to the relations given in (8).

Application of this technique requires the determination of the JONSWAP parameters $\alpha, \gamma$, and $f_{m}$ and the mean direction $\theta_{m}$. Following the approach of Hasselmann et al. [1976] and Sobey and Young [1986], $\alpha$ and $\gamma$ were represented in terms of the total spectral energy. Hasselmann et al. [1976] have presented a number of field data sets and developed the parametric relationships

$$
\alpha=0.033 \tilde{f}_{m}^{0.67}
$$

and

$$
\tilde{E}=5.28 \times 10^{-6} \tilde{f}_{m}^{-3.33}
$$

where

$$
\tilde{f}_{m}=\frac{f_{m} U_{10}}{g} \quad \tilde{E}=\frac{E g^{2}}{U_{10}}
$$

Evaluation of $\alpha$ from an equation such as (11) is complicated by the fact that due to the discrete frequency resolution of the spectral model, $f_{m}$ is not a very robust parameter. Substitution of (12) into (11), however, yields $\alpha$ in terms of Ë:

$$
\alpha=0.0029 \tilde{E}^{-0.2}
$$

The dependence of $\gamma$ on $\tilde{f}_{m}$ is less well defined, but a least squares approximation to the JONSWAP data yields 


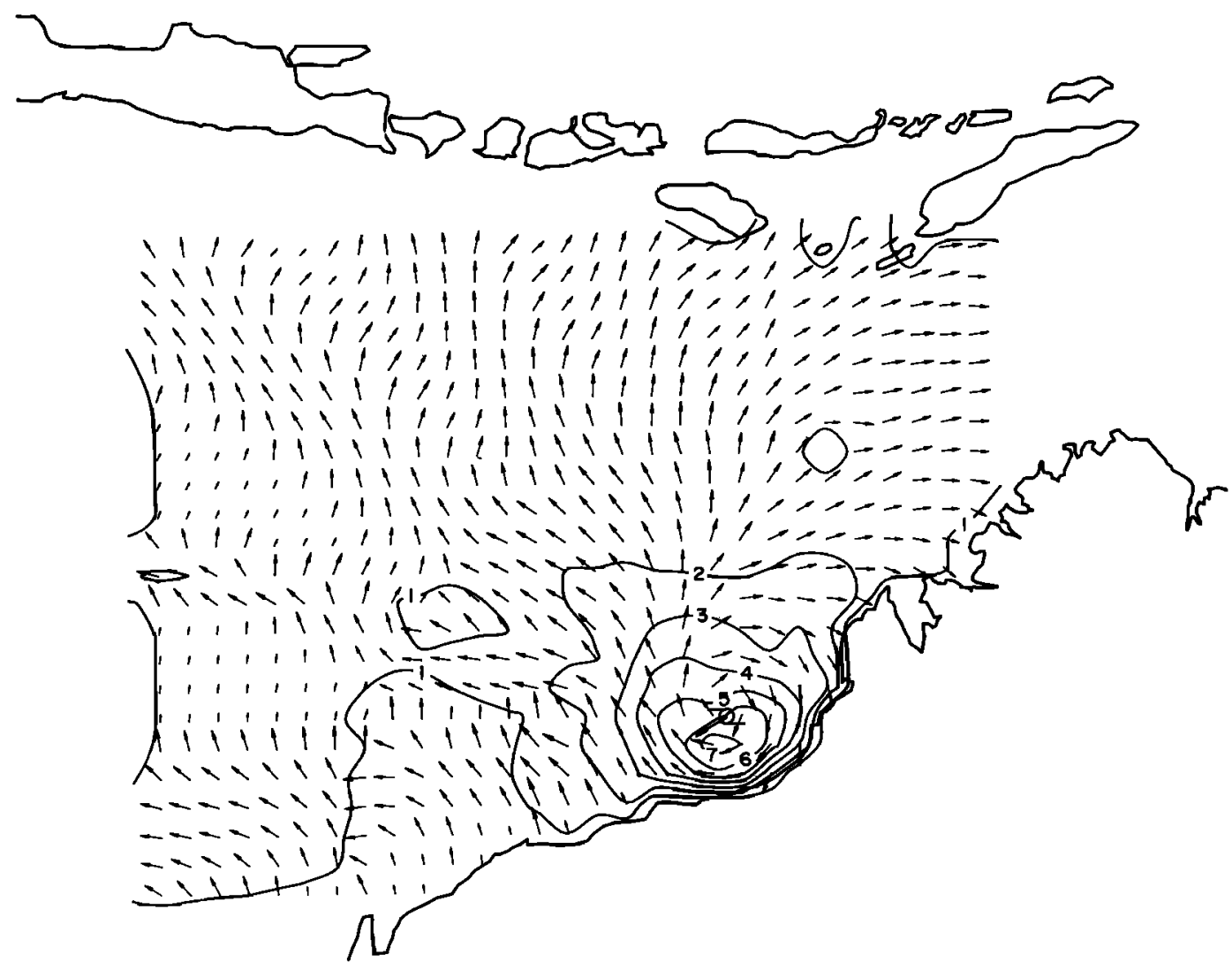

Fig. 5a. Wave field within T.C. Karen at 1200 on March 6, 1977.

$$
\gamma=4.77 \tilde{f}_{m}^{0.413}
$$

or

$$
\gamma=1.057 \tilde{E}^{-0.124}
$$

Based on the analysis of an independent data set, Mitsuyasu et al. [1980] have proposed a very similar relationship to (14): $\gamma=4.42 \bar{f}_{m}^{3 / 7}$. Equations (13) and (15) have been adopted in ADFAl. It would be expected that (11) and (14) would yield the Pierson-Moskowitz (P-M) values of $\alpha=$ 0.0081 and $\gamma=1$ for the P-M peak frequency $\tilde{f}_{m}=0.13$. This is true for (11) but not (14), where $\gamma \approx 2$ at $\bar{f}_{m}=0.13$. This result is consistent with recorded data, however. One can speculate that the final transition of $\gamma$ to the P-M value is very rapid. Such a rapid transition would result in a decrease in total spectral energy (which has not been observed) unless there is a balancing directional redistribution. Sufficient directional data at large fetch are currently not available to investigate these possibilities. Since $\gamma$ is only used in the model to scale the nonlinear transfer, this final transition is accommodated by setting $\gamma=1$ if $\bar{f}_{m} \leq 0.13$.

The determination of the peak frequency $f_{m}$ and mean direction $\bar{\theta}$ is complicated by the occurrance of spectra which do not conform to the standard JONSWAP shape. In addition, the parametric equations (13) and (15) and the nonlinear source term scaling relationships (9) and (10) are only applicable for spectra of the JONSWAP form. Typical examples where such nonconforming spectra may arise include the presence of swell and directionally skewed seas resulting from turning winds. Young et al. [1987] have indicated that for such seas the cross interactions between wind-sea and swell are not significant and, for most cases, can be considered as independent. Where wave trains are not separated significantly in direction or frequency space crossinteractions will occur. As with all second generation models, ADFA1 ignores such secondary interactions. It is assumed that the nonlinear interactions are associated with the wind-sea spectrum and hence $\bar{\theta}$ is taken as the local wind direction rather than the mean direction of the full spectrum. The spectral wind-sea peak is calculated using a weighted integral

$$
f_{m}=\frac{\int f E^{8}(f, \bar{\theta}) d f}{\int E^{8}(f, \bar{\theta}) d f}
$$

The formulation represented by (16) can produce misleading results if there are multiple peaks in the wind direction. To avoid this problem, ADFAl searches for minima in the wind direction and only carries out the integral in (16) for frequencies greater than the highest minimum. Thus swell and wind-sea contributions in the same direction are also separated.

\section{White Cap Dissipation, $S_{\text {dis }}$}

Specification of a dynamic white cap dissipation term $S_{\text {dis }}$ poses a number of problems. As with all discrete spectral 


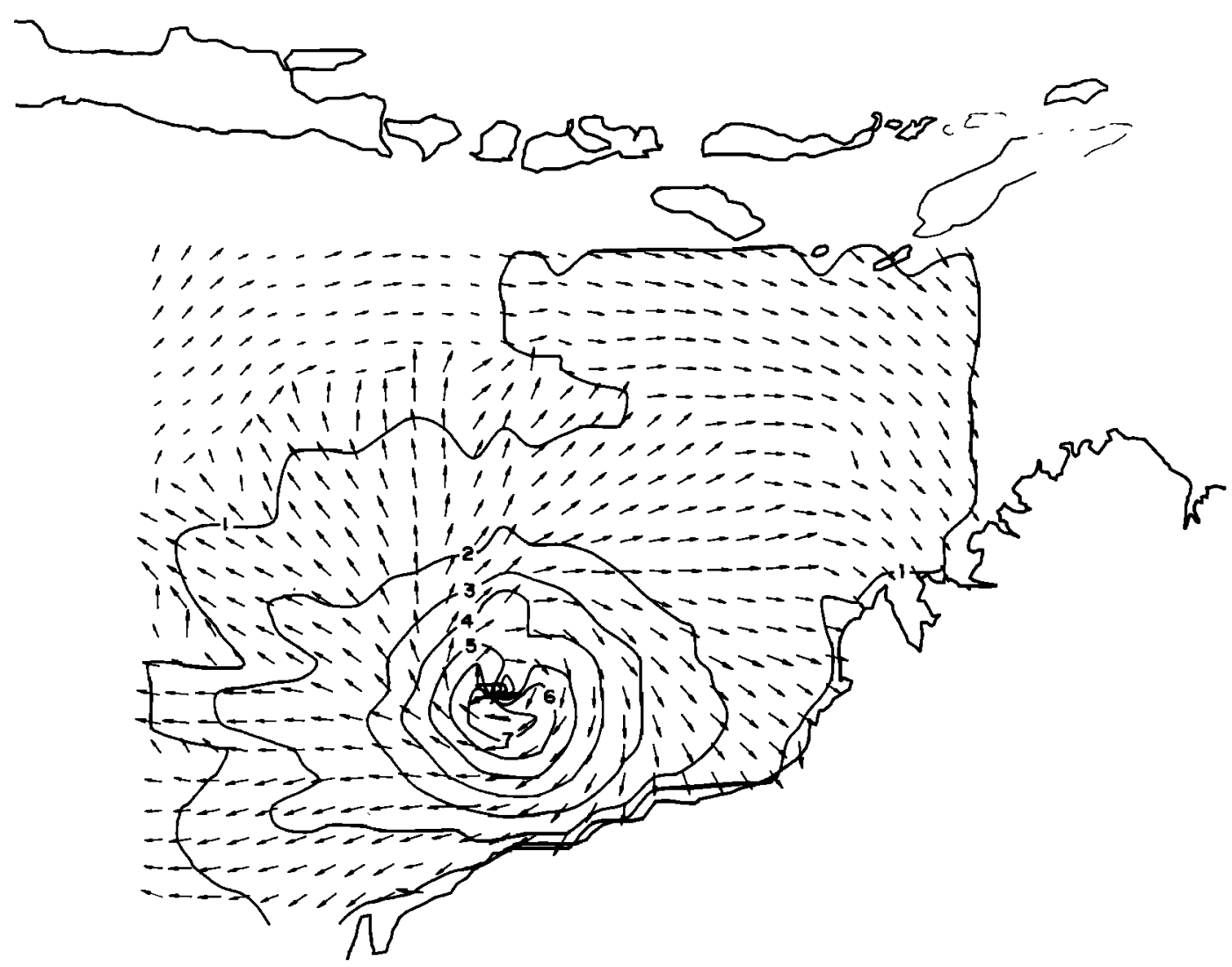

Fig. 5b. Wave field within T.C. Mabel at 1200 on January 17, 1981.

models, the wave spectrum is defined by a large number of frequency/direction bins and consequently has many degrees of freedom. Physically, local perturbations which develop in the spectrum tend to be smoothed out by the dynamic nature of the nonlinear transfer [SWAMP Group, 1985; Young et al., 1985]. The parametric representation of $S_{n l}$ used here (and in all other second generation models), however, has only a few degrees of freedom. Consequently, it cannot damp out all possible perturbations. Therefore use of this type of $S_{\text {nl }}$ term together with $S_{\text {in }}$ as represented in (7), and an analytical dissipation function $S_{\text {dis }}$ [Komen et al., 1984] will result in unstable development of the spectrum as small instabilities become unbounded. This instability problem is overcome in the present model by representing white cap dissipation by the inclusion of a saturation spectrum $E_{\infty}$ as an upper level to growth [Phillips, 1977] rather than specify an analytical form for $S_{\text {dis }}$. The saturation spectrum has the form

$$
E_{\infty}(f, \theta)=\alpha g^{2}(2 \pi)^{-4} f^{-5} D(\theta) \Phi(f, d)
$$

where $\alpha$ is the Phillips coefficient which is a function of wave age and is evaluated from (13).

$\Phi(f, d)$ is a depth dependent modification factor [Kitaigorodskii et al., 1975].

$$
\Phi(f, d)=\frac{\tanh ^{2} k d}{1+2 k d / \sinh 2 k d}
$$

and $D(\theta)$ is a directional spreading factor:

$$
D(\theta)= \begin{cases}2 / \pi \cos ^{2}\left(\theta-\theta_{w}\right) & \left|\theta-\theta_{w}\right|<90^{\circ} \\ 0 & \left|\theta-\theta_{w}\right| \geq 90^{\circ}\end{cases}
$$

If $E(f, \theta)>E_{\infty}(f, \theta)$ and $f>0.9 f_{m}$ the spectrum is reduced to $E_{\infty}(f, \theta)$. Since the saturation spectrum is centred about the local wind direction and is only applied to components at frequencies higher than the wind-sea peak, it has no influence on the propagation of swell. The saturation level is only included to bound the actively growing windsea portion of the spectrum. Phillips [1985] has indicated that a more consistent formulation for the saturation spectrum is $E_{\infty}(f) \propto u_{*} g f^{-4}$ rather than $E_{\infty}(f) \propto g^{2} f^{-5}$ as indicated by (17). For practical wave prediction the difference between the two formulations is of little consequence. In view of this and the weight of field data which have been used to determine the proportionality factor in (17), this form has been retained. In addition, the nonlinear source term scaling relationship (9) is only applicable for spectra with an $f^{-5}$ high-frequency face. Therefore use of (17) ensures the applicability of (9).

\section{Bottom Friction, $S_{\mathrm{bf}}$}

In water of finite depth, waves are influenced by bottom friction and begin to decay. Following Hasselmann and 


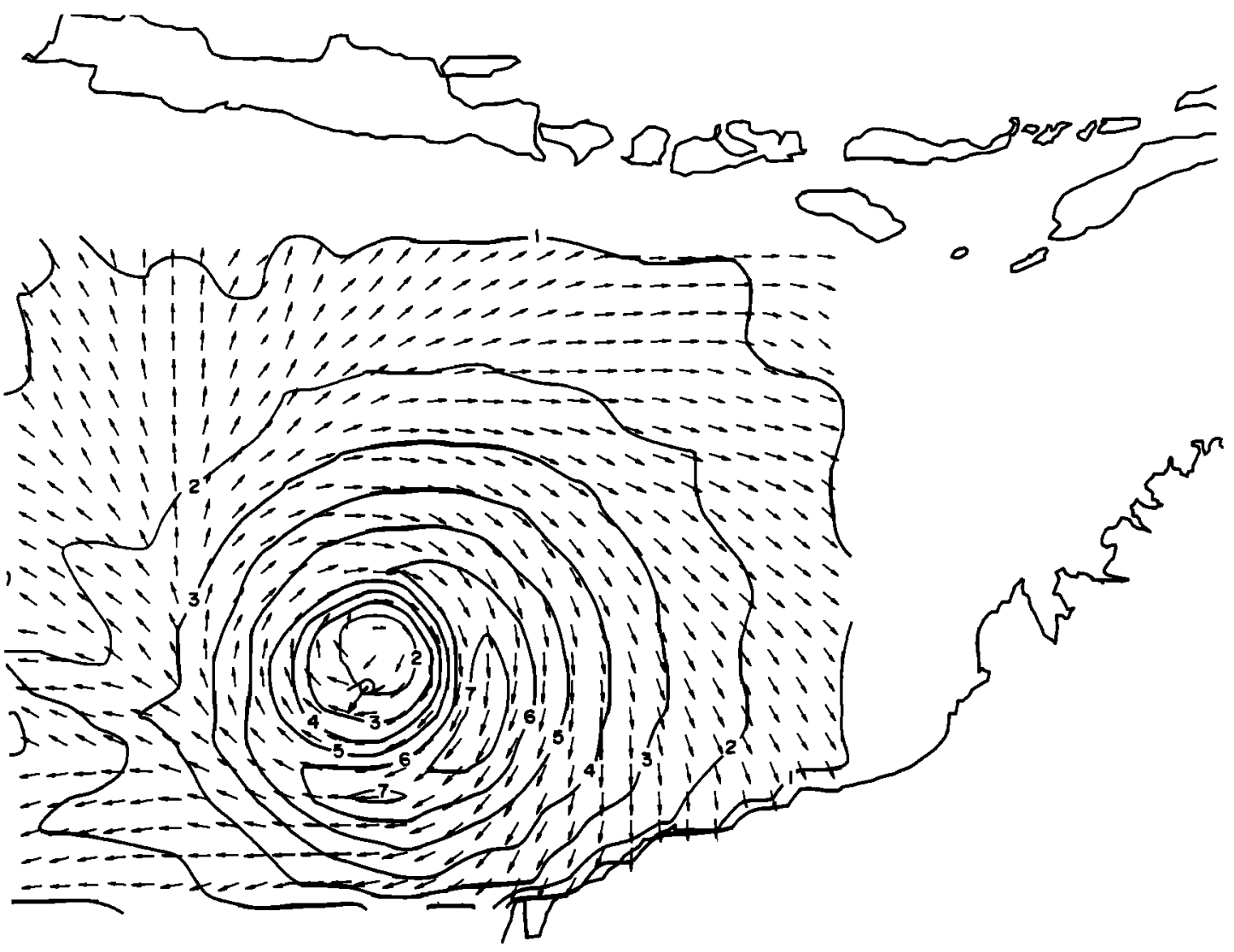

Fig. 5c. Wave field within T.C. Alby at 1200 on March 30, 1978.

Collins [1968], the source term representing bottom friction is of the form

$$
S_{\mathrm{bf}}(f, \theta)=-\frac{C_{f} g k^{2} u_{b}}{\omega^{2} \cosh (k d)} E(f, \theta)
$$

where $u_{b}$ represents the wave-induced bed velocity

$$
u_{b}^{2}=g^{2} \int \frac{E_{1}(f)}{C^{2} \cosh ^{2}(k d)} d f
$$

where $E_{1}(f)$ is the one-dimensional spectrum. The friction factor $C_{f}$ can be varied if information is available for a particular area, but a value of 0.01 has proved successful under a variety of applications.

\section{Depth Limited Breaking, $S_{\mathrm{brk}}$}

The white cap dissipation term $S_{\text {dis }}$ discussed earlier represents the processes by which energy is lost through wave breaking in the high-frequency portion of the spectrum. In addition, as waves propagate into shallow water, the effects of shoaling lead to an increase in energy, this increase being greater at lower frequencies. Eventually, such waves become depth limited and begin to break. This process should not be confused with the white capping represented by $S_{\text {dis }}$. The processes associated with depth-limited breaking are still largely unknown, but considerable work has been con- centrated on determining the limiting wave height. Divoky et al. [1970] and Chen and Wang [1983] have represented the limiting steepness of waves by the relationship

$$
\frac{H_{b}}{L}=0.12 \tanh k d
$$

where $H_{0}$ is the significant wave height and $L$ the wavelength. Reformulating (22) in terms of integral properties of the spectrum yields

$$
\bar{E}_{\max }=\frac{0.0355 \bar{C}^{4}}{g^{2}}
$$

where $\bar{E}=\int E(f, \theta) d f d \theta$ and $\bar{C}$ is the phase speed of the mean frequency of the spectrum.

Equation (23) places a limit on the total energy of the spectrum but gives no information as to the limiting spectrum itself. As long waves "feel" the bottom to a greater extent than short waves, depth-limited wave breaking would be expected to occur first at low frequencies, moving progressively to higher frequencies with decreasing depth. Within ADFA1, if the total spectral energy exceeds that given by (23), the energy is reduced by successively removing energy from low frequencies until the $\bar{E}_{\max }$ limit is reached.

It is interesting to investigate the asymptotic limits of (23). For shallow water, $\bar{C}=\sqrt{g d}$, and (23) becomes 


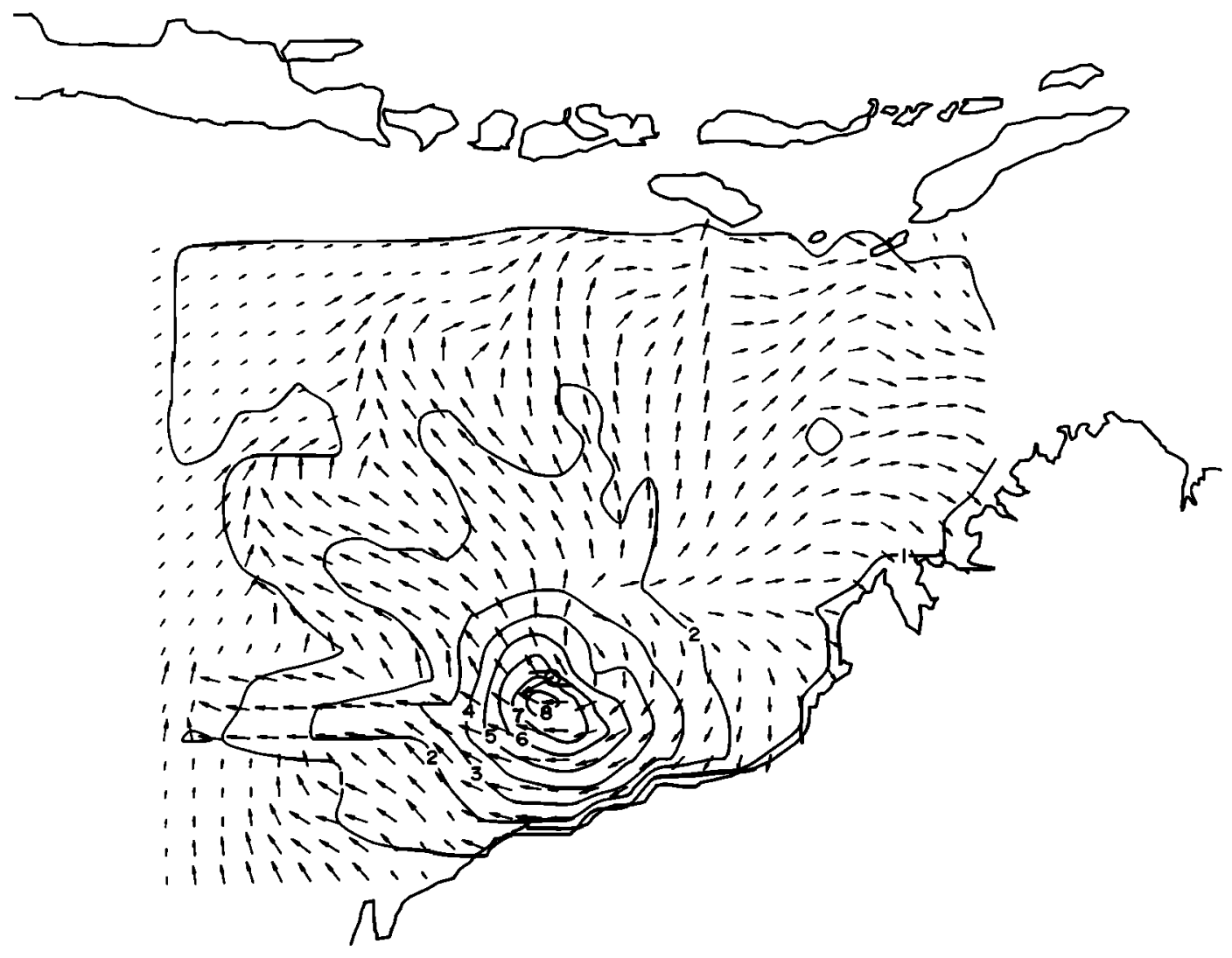

Fig. 5d. Wave field within T.C. Madge at 1200 on March 11, 1973.

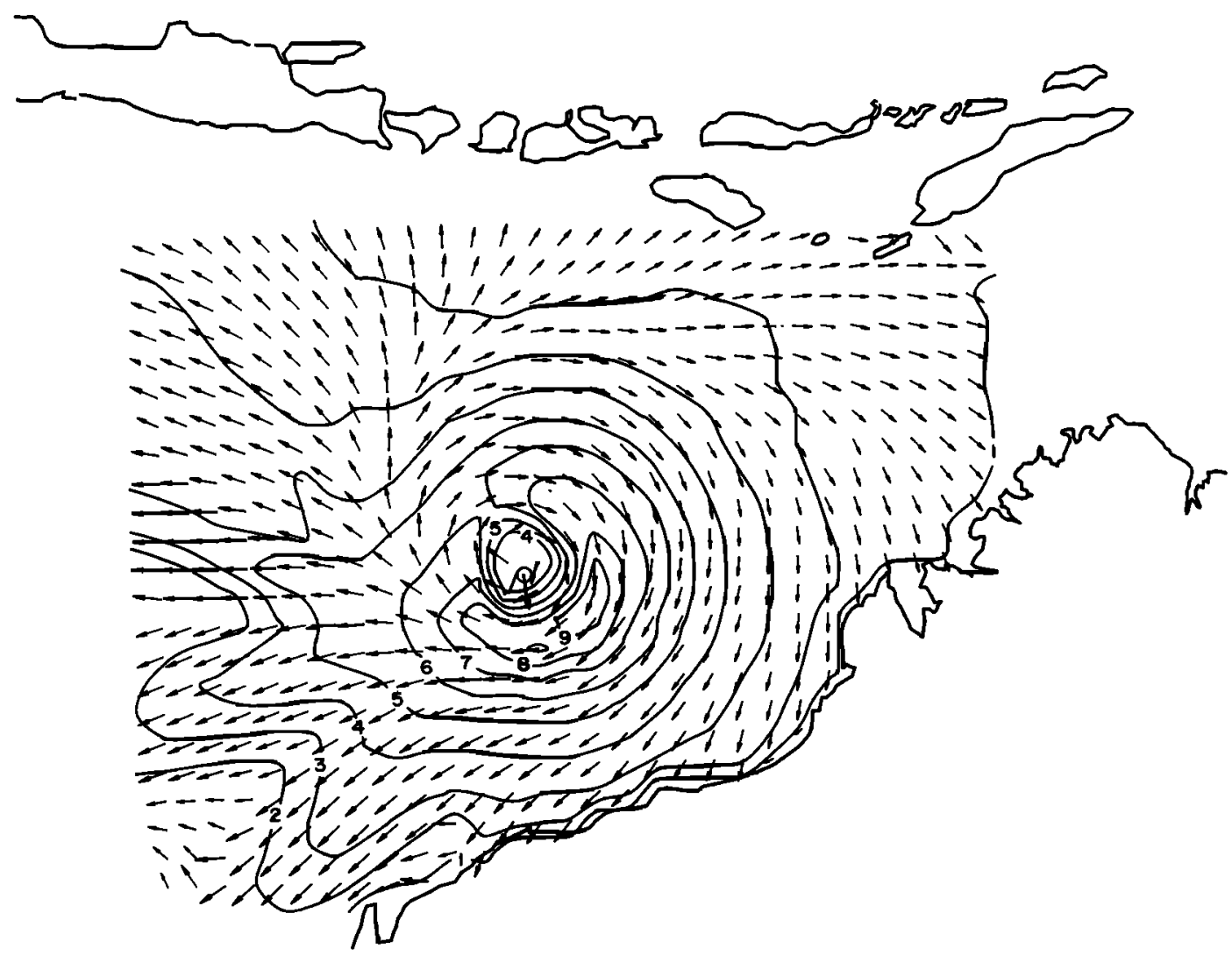

Fig. 5e. Wave field within T.C. Dean at 1200 on January 31, 1980. 

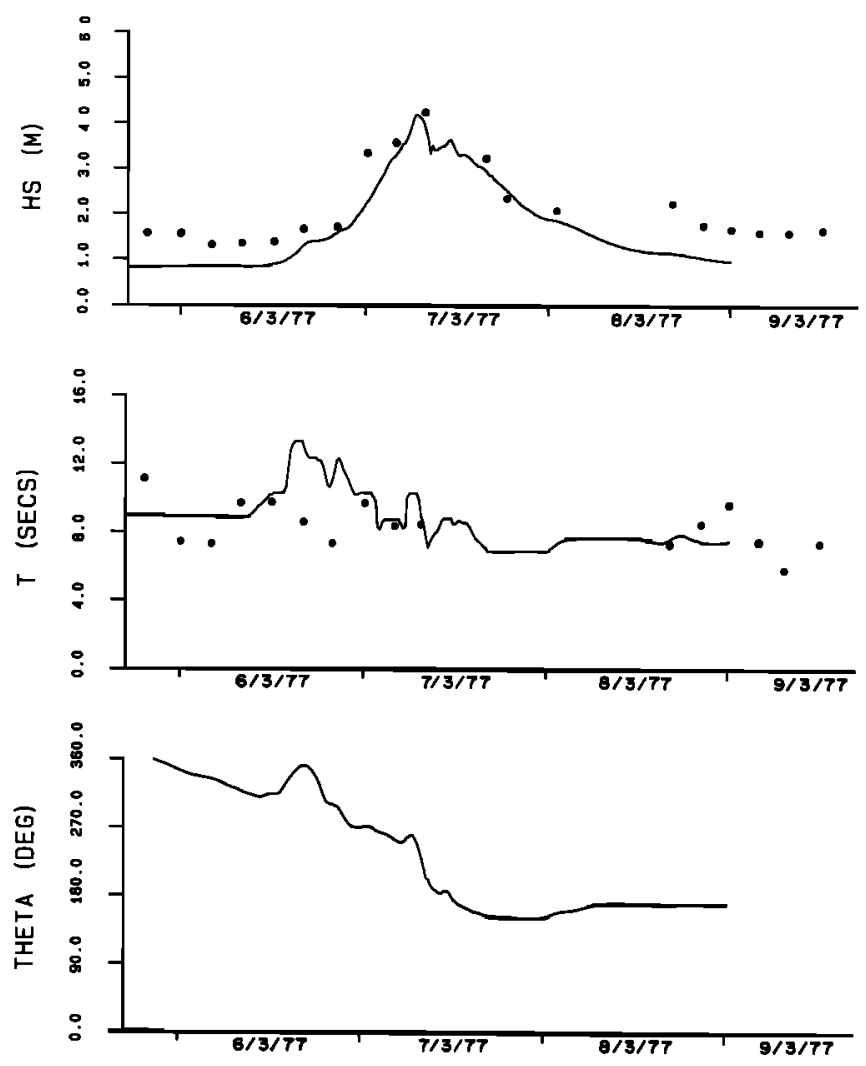

Fig. 6a. Comparison between recorded data and model predictions during T.C. Karen at North Rankin.

$$
\bar{E}_{\max }=0.0355 d^{2}
$$

or $H_{0}=0.75 d$, very close to the $0.78 d$ limit commonly used. For deep water $\bar{C}=g /(2 \pi f)$, and (23) yields $H_{s}=0.12 L$, where $L$ is the wavelength. This value corresponds to the commonly used limiting deep-water wave slope of 0.14 .

\section{Numerical Solution}

Equation (1) can be solved numerically in one of three fashions: finite difference/element methods, full ray methods, or piecewise ray methods. In shallow water applications the bending of wave rays described by (2) implicitly accounts for the effects of refraction, thus favoring use of the ray techniques. Full ray methode construct rays which propagate throughout the complete grid. Equation (1) is then integrated numerically along these rays. This process decouples the rays, thus making it difficult to use source term representations requiring knowledge of the full spectrum such as nonlinear interactions and bottom friction. Piecewise ray methods trace all wave ray components from each computational node backward for one time step. The energy at each terminating ray point is then determined by interpolation amongst surrounding nodal points. Since the full spectrum is always available at each grid point, the individual spectral components can now be coupled through mechanisms such as nonlinear interactions.
ADFA1 uses a fractional step solution [Sobey and Young, 1986] in which propagation and forcing are conducted over alternate half time steps. The propagation step uses piecewise rays in conjunction with a third-order spatial interpolation scheme to reduce the effects of numerical dispersion [Young, 1987a].

The prediction of wave spectra in shallow water involves a conflict of scales. To represent accurately the influences of shallow water, a fine grid which adequately defines the bathymetry is required. To represent the propagation of energy into shallow water regions, however, a geographically large area must be modeled. To accomplish these requirements and still maintain acceptable computational expense, ADFA1 uses a system of subgrids. A large course grid is used in deep water. Nested within this grid can be any number of progressively finer grids.

Boundary conditions for the course grid are generally of the radiation type, in which energy can leave the grid but none can enter. The use of such a condition is valid provided there are no major generation areas outside the grid. Consequently, the course grid must be sufficiently large to include all possible generating areas. The nested finer grids then use their respective coarser grids to provide boundary values.

\section{Comparison With Recorded Data}

The SWAMP Group [1985] intercomparison of spectral wave models has provided a consistent set of deep water
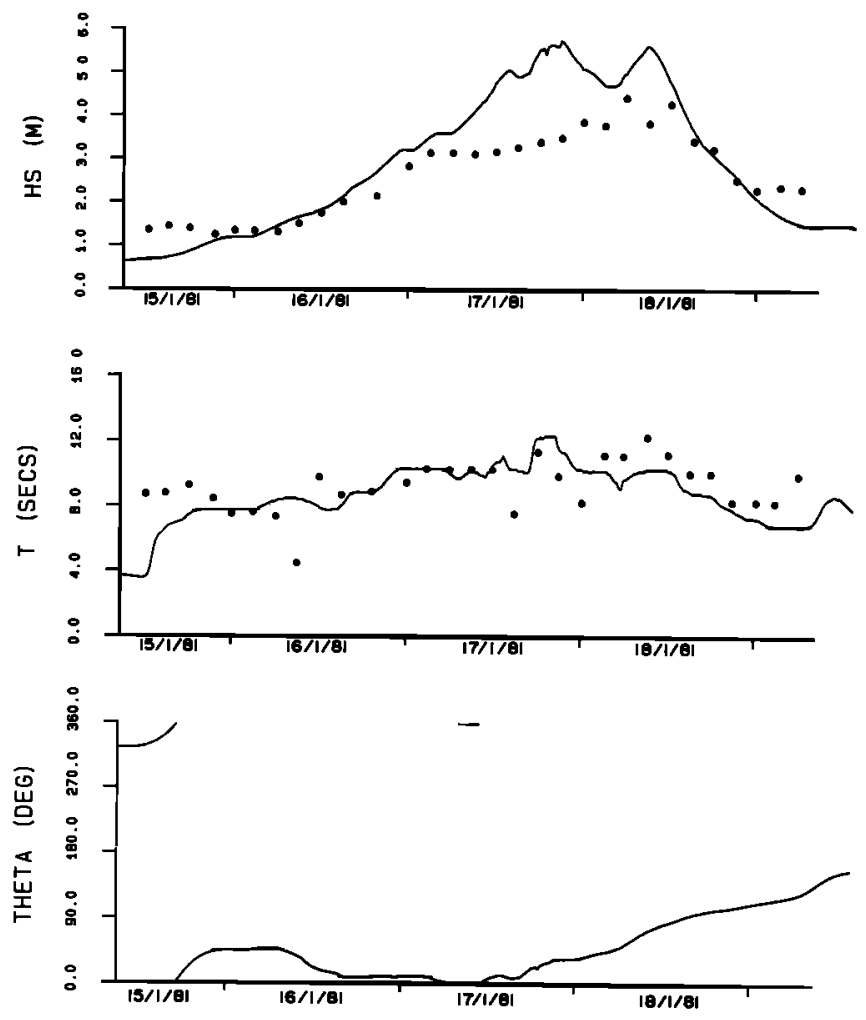

Fig. 6b. Comparison between recorded data and model predictions during T.C. Mabel at North Rankin. 

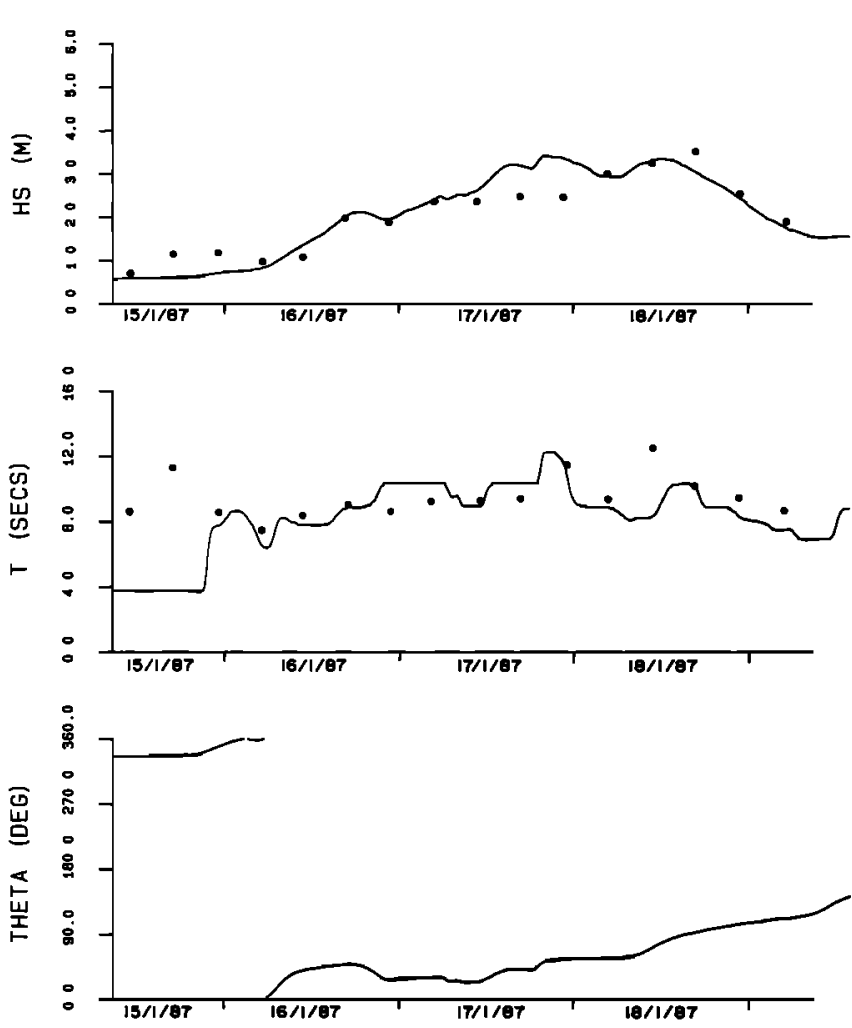

Fig. 6c. Comparison between recorded data and model predictions during T.C. Mabel at Legendre Island.

tests for the evaluation of model performance. Due to space limitations, these results are not presented here. Detailed results for all SWAMP tests are, however, presented by Young [19876].

As demonstrated by SWAMP Group [1985], tropical cyclone conditions provide a very demanding test of model performance. This occure due to the rapid spatially varying winds which occur. The ideal opportunity for an intercomparison under such conditions was provided by Woodside Offshore Petroleum Pty Ltd., the operators of an offshore gas field and associated port facility on the northwest coast of Australia. Data have been collected in this region under tropical cyclone conditions for a period of approximately 15 years. The very extensive data base generated by this program includes data from a variety of sites encompassing both deep and shallow water and hence provides the ideal means for model validation.

For all storms, meteorological data including central pressure, track, and radius to maximum winds were estimated from Australian Bureau of Meteorology analyses. It should be noted that the intercomparisons which follow are intended to provide a validation of the model physics as previously described. No attempt has been made to calibrate the model against recorded data.

As discussed in section 4, a system of subgrids was used for the numerical modeling. Three grids as shown in Figure 3 were found to be adequate, the space and time steps reducing by a factor of 5 between each grid. Although numerical simulations have been performed for the majority of the storms within the data base, space limitations restrict the number which can be presented. Five tropical cyclones which are typical for the region have been selected, and their tracks shown in Figure 4. Tropical cyclone tracks for the region are very erratic but can be grouped into three major categories. The majority of the storms move from ENE to WSW, roughly parallel to coast as illustrated by cyclones Karen, Madge and Alby. The remainder develop in the NW and move toward the coast. Such storms either continue on this track and cross the coast, as illustrated by Dean or curve to the south, similar to Mabel.

Figure 5 shows typical examples of the spatial distributions of significant wave height $H_{s}$, peak wave period $T_{m}=$ $1 / f_{m}$, and mean wave direction $\theta_{m}$. Each of the figures has been taken at the approximate height of the respective storms and is depicted on the large scale A grid (see Figure 3). The significant wave height $H_{0}$ is represented by contours with a superimposed vector field, the length of which are proportional to $T_{m}$ and the direction representing $\theta_{m}$. Tropical cyclones (T.C.) Mabel, Alby, and Dean clearly show the crescent-shaped region of high waves to the left of the storm center as reported by numerous authors and expected due to the asymmetry of the wind field. The wave field in Karen and Madge is distorted due to the storms' proximities to land. In all cases, the vector pattern shows that paricularly in the regions ahead of the storm, the wave field is dominated by swell radiating out from the intense generation region near the center. Almost identical patterns have also been observed in tropical cyclones using synthetic aperture radar [King and Shemdin, 1978; Gonzalez et al.,
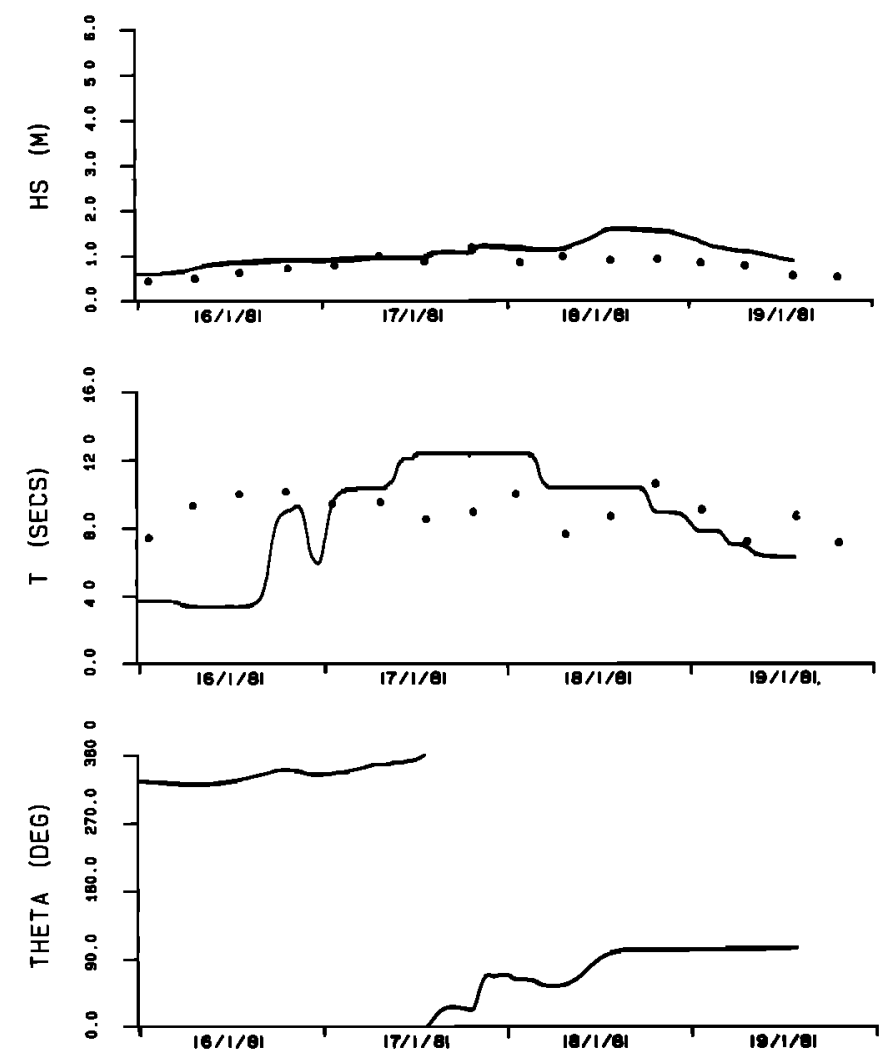

Fig. 6d Comparison between recorded data and model predictions during T.C. Mabel at Withnell Bay. 

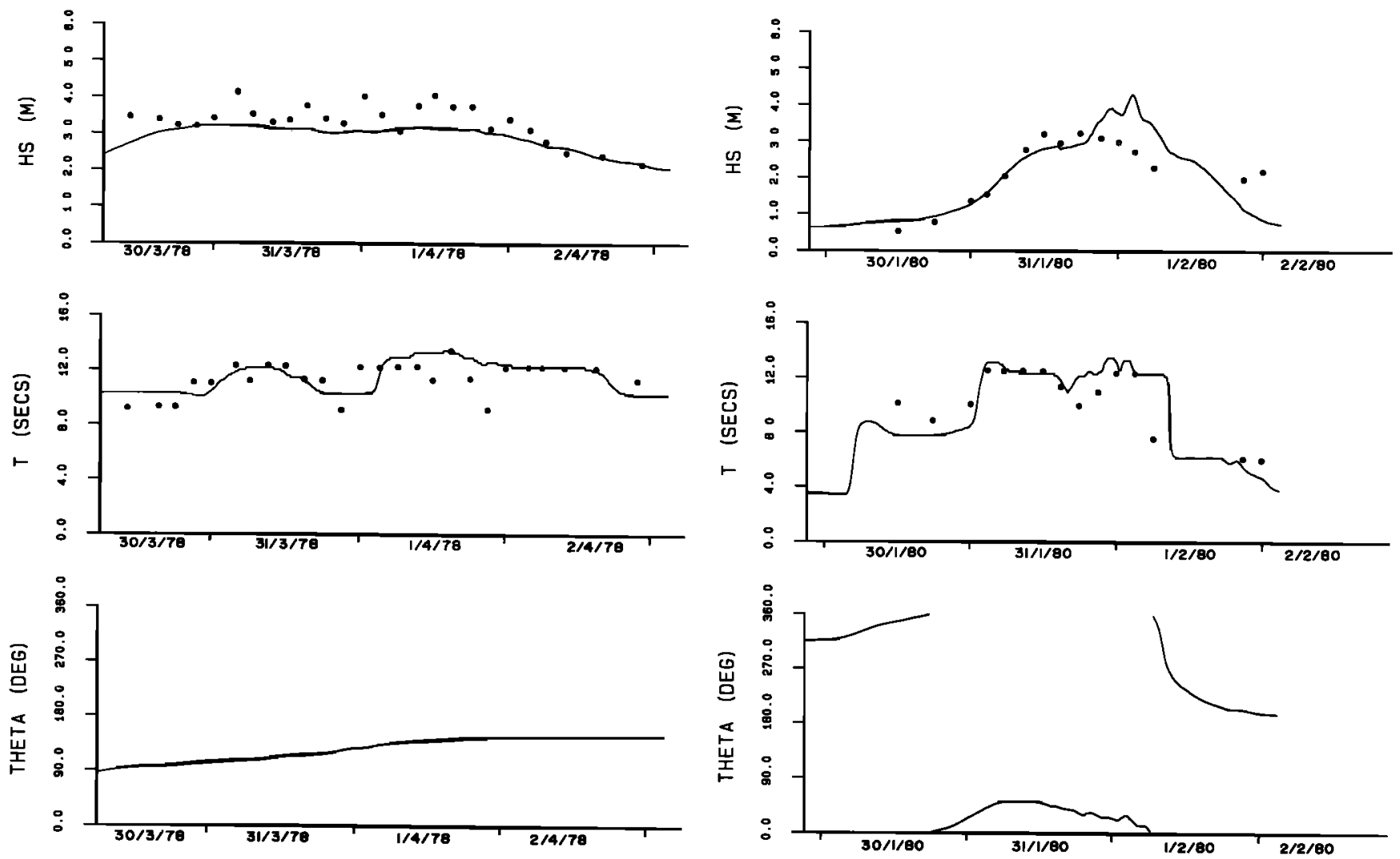

Fig. 6e. Comparison between recorded data and model predictions during T.C. Alby at North Rankin.

Fig. 6g. Comparison between recorded data and model predictions during T.C. Dean at Legendre Island.
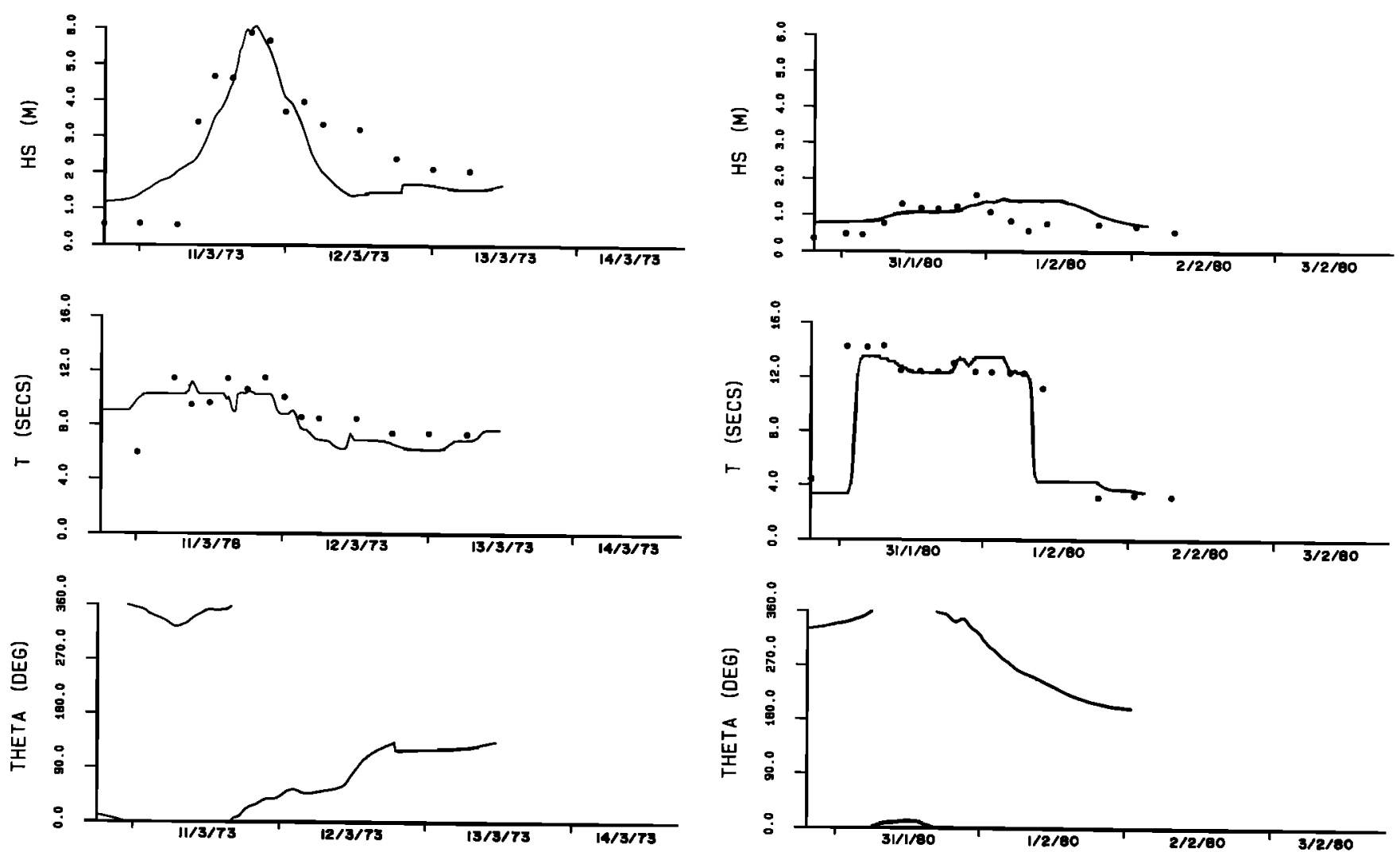

Fig. 6f. Comparison between recorded data and model predictions during T.C. Madge at North Rankin.

Fig. 6h Comparison between recorded data and model predictions during T.C. Dean at Withnell Bay. 


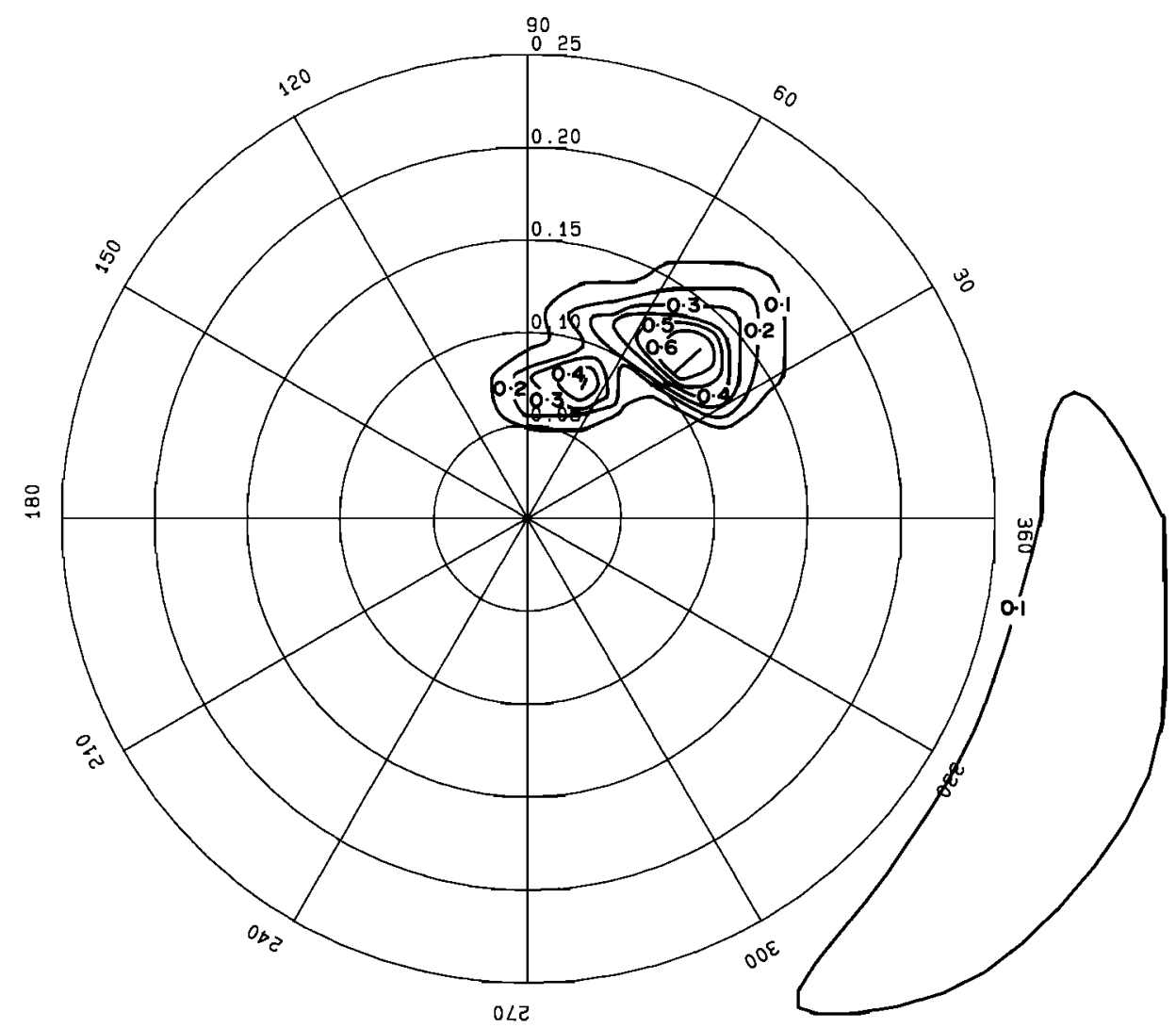

Fig. 7a. Predicted directional wave spectrum at Legendre Island on January 31, 1980, at 0000.

1982; McLeish and Ross, 1983; Holt and Gonzalez, 1986]. In a number of the figures a sharp demarcation can be seen approximately $1000 \mathrm{~km}$ ahead of the storm, indicating the extent to which swell has propagated. The exact position of this front will depend on the difference between the forward speed of the storm and the group velocity at which the swell propagates.

Recorded data from wave rider buoys were available for each of these storms, although the actual position and number of instruments varied for each event. The various data recording locations are shown in Figure 3. Figure 6 shows comparisons between recorded and predicted values of $H_{s}$ and $T_{m}$ at the available data locations during the passage of each storm. In addition, predicted values of $\theta_{m}$ are also presented, although no directional data were available for comparative purposes.

T.C. Karen was a moderately intense storm with a minimum central pressure of 971 mbar. It moved parallel to the shore and passed approximately $40 \mathrm{~km}$ east of the deepwater recording site at North Rankin (depth $120 \mathrm{~m}$ ). Figure $6 a$ indicates good agreement between model and recorded data for both $H_{s}$ and $T_{m}$. The model tends to under predict in the early and latter stages of the storms passage but agrees well during the height of the storm. This occurs as a result of the relatively simple wind field used, which assumes that the tropical cyclone vortex exists in isolation. In reality, the cyclone will be embedded in a background flow. Consequently, the wind field model will tend to underestimate the wind speed and hence $H$, when the storm is remote from the recording site. A more sophisticated wind field is currently being investigated. The good agreement for $T_{m}$ is very impressive when it is remembered that this is not a particularly robust parameter. Under tropical cyclone conditions, where the spectrum often consists of both wind-sea and swell, this parameter may fluctuate markedly.

During the passage of T.C. Mabel data were recorded at North Rankin (120 m), Legendre Island (36 m), and Withnell Bay $(8 \mathrm{~m})$. The storm, which was relatively intense moved first toward the coast and then turned south to continue parallel. Although the closest approach to North Rankin was $190 \mathrm{~km}$, a maximum significant wave height of approximately $5 \mathrm{~m}$ was recorded. Agreement between model and recorded data (Figure $6 b$ ) is again good, except in the early and late stages of the simulation, as discussed earlier. Conditions at the shallower site at Legendre Island (Figure $6 c$ ) are very similar to those farther offshore, indicating that little shallow water attenuation has occurred. At Withnell Bay (Figure 6d), however, which is afforded protection by coastal features, there is considerable attenuation with a maximum $H_{a}$ of approximately $1 \mathrm{~m}$. Again the model performs well, although it indicates slightly greater values of $T_{m}$ than recorded. This indicates more long-period swell entering Mermaid Sound and hence Withnell Bay than recorded. As illustrated in Figure 5, swell tends to radiate out from the center of the storm. Therefore such small disagreements could be the result of slight errors in the storm track.

T.C. Alby came no closer than $550 \mathrm{~km}$ from North Rankin when its central pressure was 990 mbar. Despite the re- 


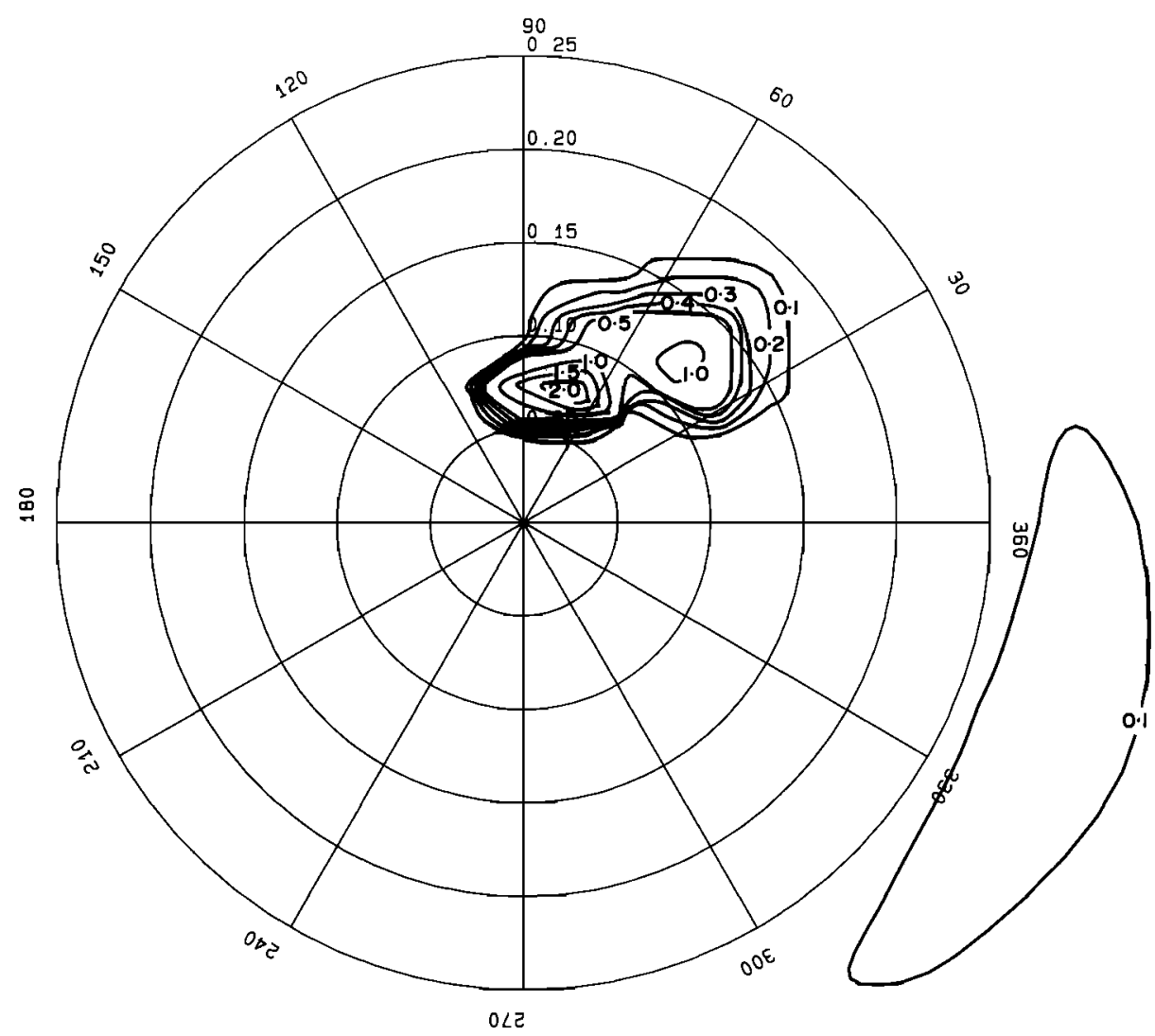

Fig. 7b. Predicted directional wave spectrum at Legendre Island on January 31, 1980, at 0300

moteness of the storm, quite severe wave conditions with a maximum $H_{s}$ of approximately $4 \mathrm{~m}$ were recorded (Figure $6 e$ ). In addition, high wave conditions persisted for a number of days. These features are a result of the very large spatial extent of the storm, as can be seen in Figure $5 c$. Again the agreement between model and recorded data is quite good, although the model consistently underpredicts $H_{\text {o by between }} 0.5 \mathrm{~m}$ and $1.0 \mathrm{~m}$. This is possibly due to the problems of accurately positioning the center of such a large storm and the inadequacy of the wind field model for such a remote storm.

T.C. Madge was very similar to Mabel, although its track was simpler, moving parallel to the coast. Like Mabel it passed approximately $190 \mathrm{~km}$ seaward of North Rankin and generated a maximum $H_{s}$ of $6 \mathrm{~m}$ (Figure 6f). Again agreement between model and recorded data is quite good.

T.C. Dean has a significantly different track to the storms mentioned earlier, approaching almost normal to the coastline and making land fall approximately $200 \mathrm{~km}$ north of the recording sites at Legendre Island and Withnell Bay. Wave conditions at Legendre Island peak at $H_{0} \approx 3.5 \mathrm{~m}$ (Figure 6g), whereas within Mermaid Sound at Withnell Bay this has been reduced to approximately $1 \mathrm{~m}$ (Figure $6 h$ ). At both sites, agreement between model and recorded data is very good. In particular, the behavior of $T_{m}$ is very interesting. Both model and recorded data at both sites indicate a sudden increase in $T_{m}$ at 0200 on January 31, 1980, marking the arrival of swell and its subsequent pen- etration into Mermaid Sound. These conditions continue until 0800 on February 1, 1980, when the swell abruptly stops. Examination of the directional spectra at Legendre Island as predicted by the model (Figure 7) clearly show that the sea is composed of low-frequency swell from NE and a quite independent locally generated wind-sea from SE. Between 0000 and 0300 on January 31, 1980, the magnitude of the swell peak increases considerably as shown in Figure 7b. A similar situation also exists at Withnell Bay (Figure 8) although here the swell is from the north. This occurs as a result of both refraction and also the protection provided by Burrup Peninsula which prevents much of the swell penetrating into the region. Comparisons of the model and recorded one-dimensional spectra at Withnell Bay are shown in Figure 9. Again the agreement is very impressive with the model values falling well within the $90 \%$ confidence limits on the recorded data. As the cyclone moves closer to shore, the point is eventually reached when swell radiating from the center can no longer reach the recording sites. This accounts for the sudden decrease in $T_{m}$ at 0800 on February 1,1980 .

A good indication of overall model performance is demonstrated in Figure 10 which shows a composite comparison at all recording sites for the five storms mentioned earlier. Figure 10 shows all data in the period plus or minus 24 hours of the maximum recorded value of $H_{a}$ for each storm. Statistical analysis of this data yields an average error of -8 $\mathrm{cm}$, an rms error of $46 \mathrm{~cm}$ and a scatter index of $15 \%$. Fol- 


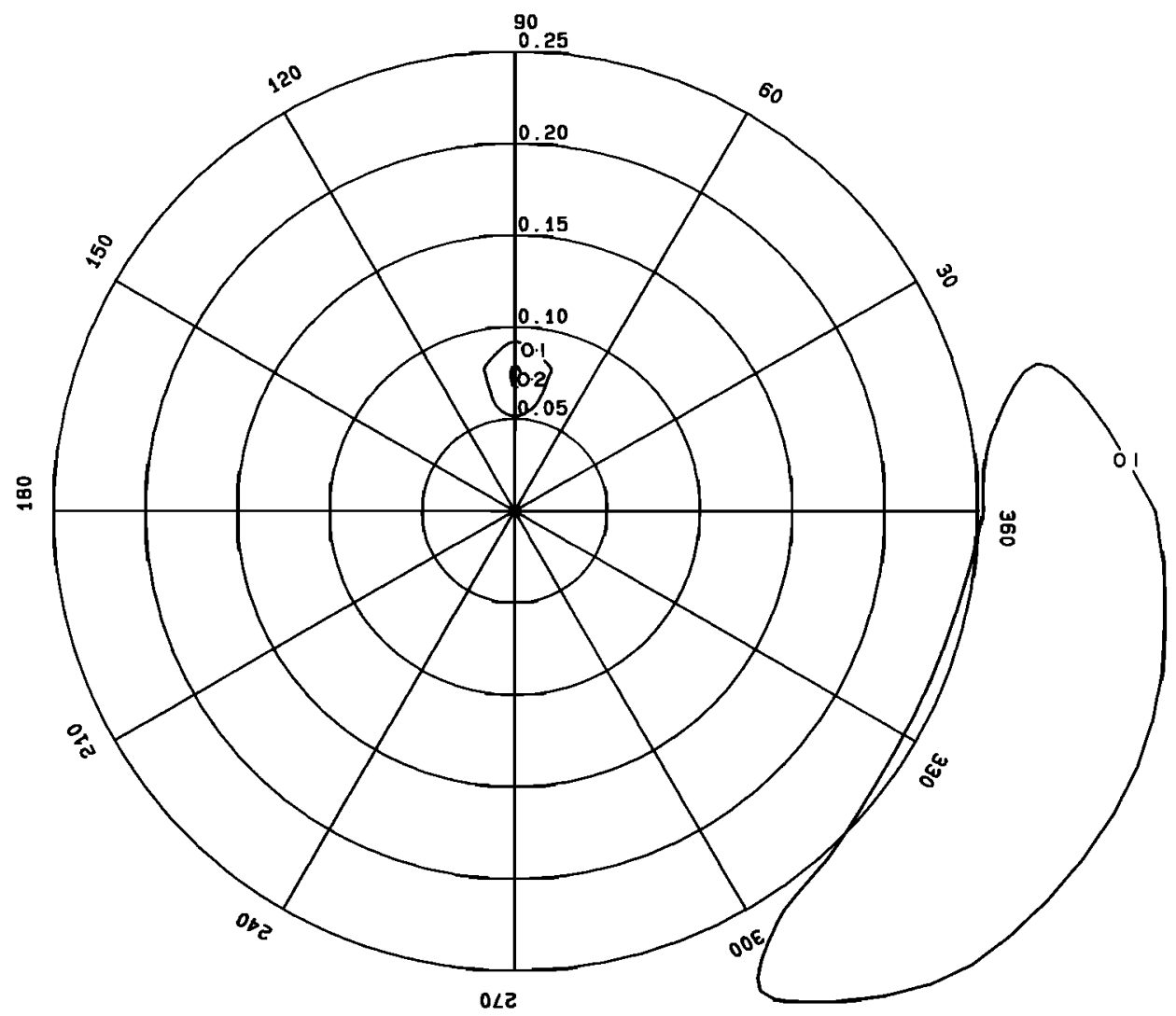

Fig. 8a. Predicted directional wave spectrum at Withnell Bay on January 31, 1980, at 0000 .

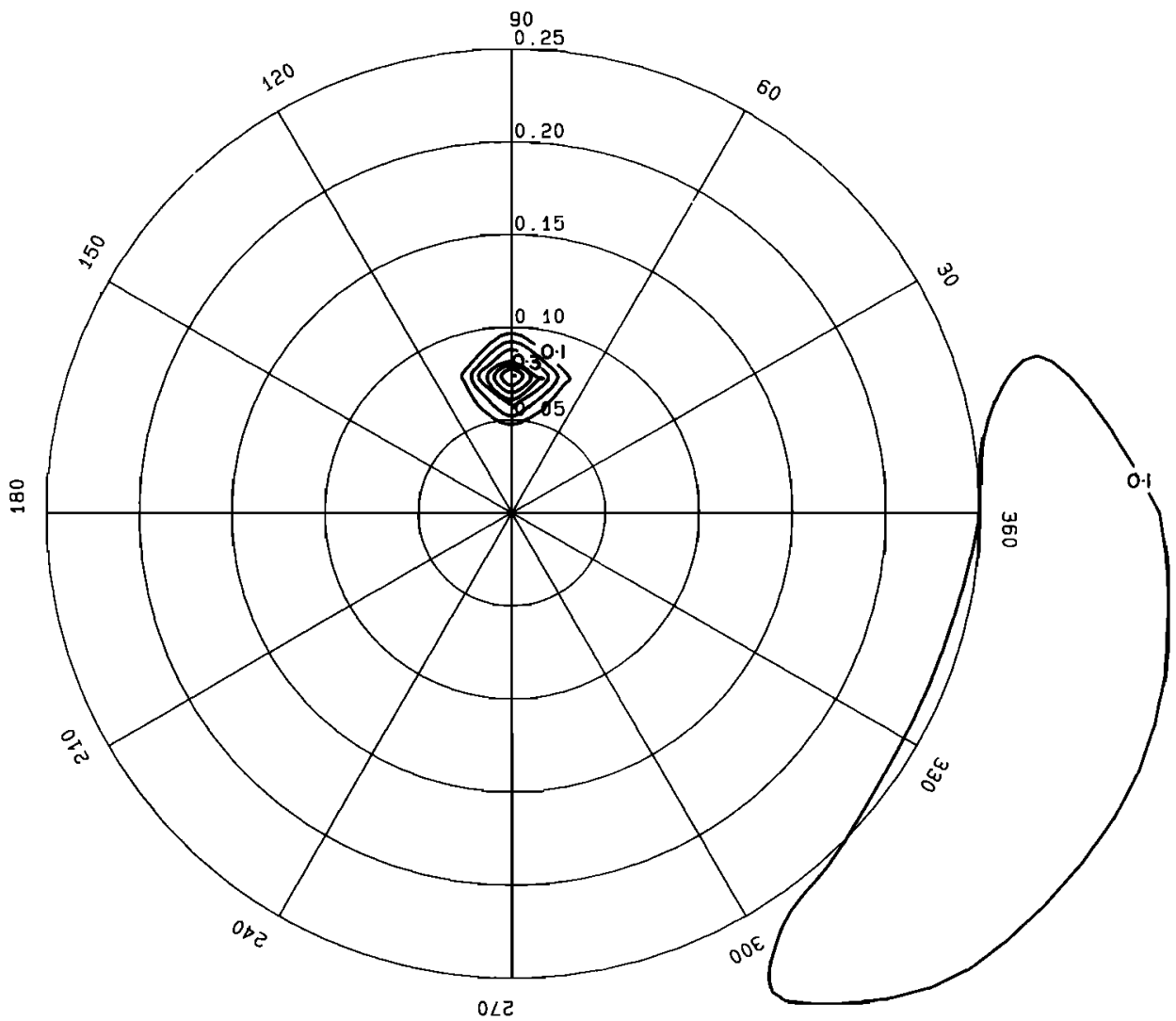

Fig. 8b. Predicted directional wave spectrum at Withnell Bay on January 31, 1980, at 0300 . 


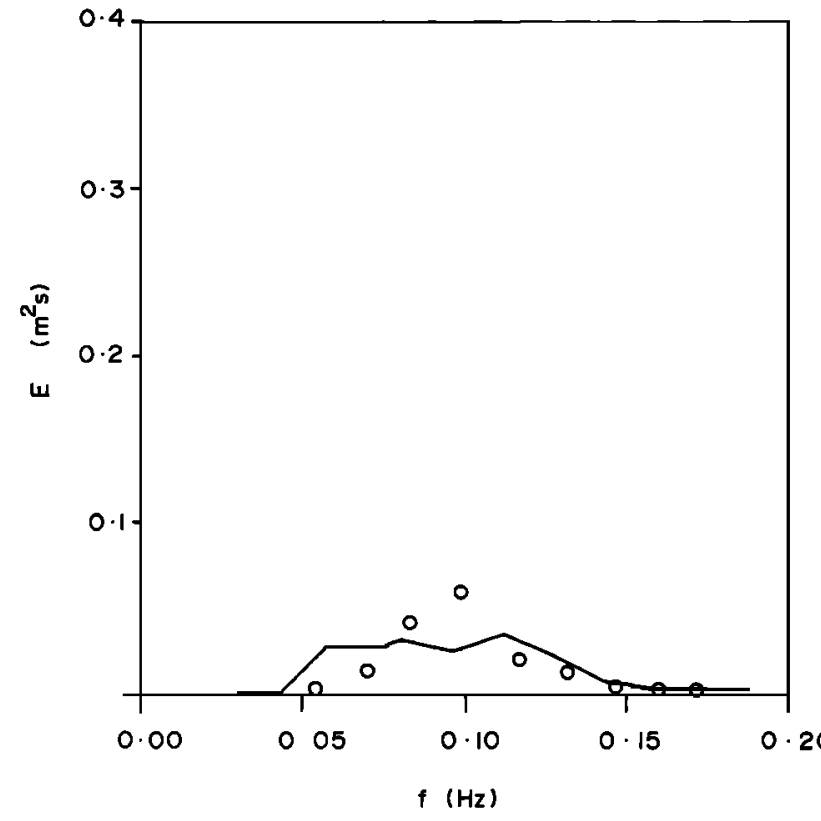

Fig. 9a. Comparison between recorded and predicted onedimensional spectra at Withnell Bay on January 31, 1980, at 0000 .

lowing Günter et al. [1984], the average error is defined as the mean of the modeled minus observed $H_{s}$ and the scatter index as the ratio of the standard deviation and mean observed $H_{\theta}$. Agreement between observed and model results appear to be better for the larger wave heights. This is due to the more accurate representation of the wind field by the simple vortex model when the storms are relatively close to the recording sites, as discussed earlier. In view of possible

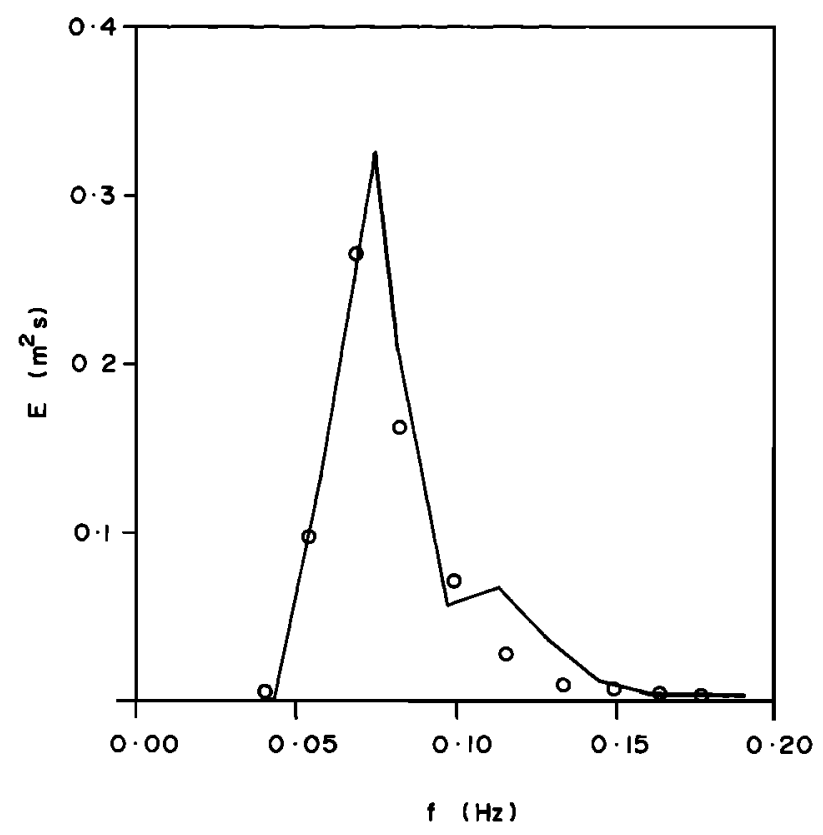

Fig. 96. Comparison between recorded and predicted onedimensional spectra at Withnell Bay on January 31, 1980, at 0300 .

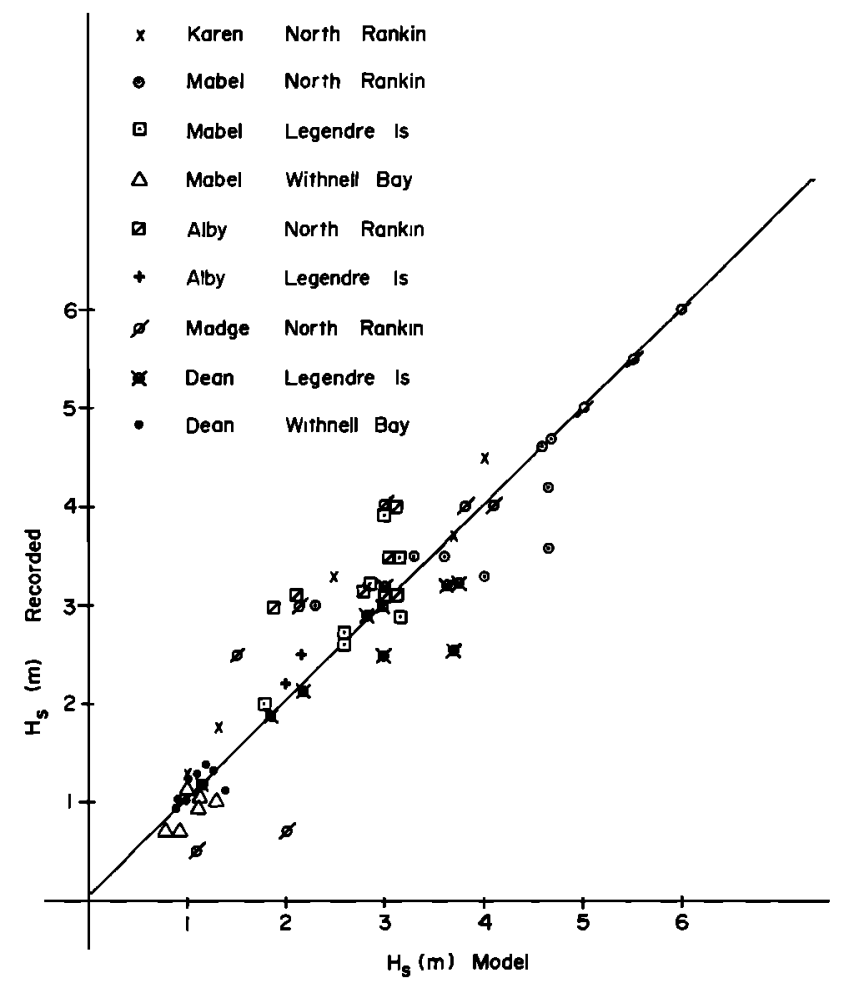

Fig. 10. Composite comparison of recorded and predicted values of significant wave height from all storms at both deep and shallow water locations.

errors in accurrately positioning the storm tracks and meteorological parameters, the agreement is very impressive. In particular, model performance in shallow water appears to be equally good as that in deep water.

\section{Conclusions}

A numerical wave prediction model has been presented which is based on solution of the radiative transfer equation. The model adopts a simple yet very flexible representation of the nonlinear source terms in this equation. This form of approximation appears to perform quite well under the very demanding conditions produced by the turning winds of tropical cyclones.

A very extensive comparison with recorded data, both in deep and shallow water, under tropical cyclone forcing clearly illustrates model performance under very demanding meteorological conditions. The model reproduces observed directional trends in tropical cyclones with the dominant swell propagating ahead of the storm. In addition, the model consistently provides good agreement with recorded data for significant wave height and peak wave period.

Acknowledgments. The bulk of the development of ADFA1 was performed within the Department of Civil Engineering at the Australian Defence Force Academy. Early development of the model, however, was conducted while the author was employed as a Queen's Fellow within the Department of Civil and Systems Engineering, James Cook University of North Queensland. The financial support provided by the Marine Research Allocations Advisory Committee is gratefully acknowledged. The author also wishes to thank the management of Woodside Offshore Petroleum Pty. Ltd. and the North West Shelf Joint Venture Participants for making available the tropical cyclone data presented in the pa- 
per. The participants in the LNG phase of the project are Woodside Petroleum Pty. Ltd., BHP Petroleum Pty. Ltd., BP Developments Australia Ltd., California Asiatic Oil Company, Japan Australia LNG (MIMI), and Shell Development (Aust) Pty. Ltd.

\section{REFERENCES}

Allender, J.H., T.P. Barnett and M. Lybanon, The DNS model: An improved spectral model for ocean wave prediction, in The Sea Wave Modeling Project, SWAMP Group, Plenum, pp. 235248, New York, 1985.

Barnett, T.P., On the generation, dissipation and prediction of ocean wind waves, J. Geophys. Res., 79, 513-529, 1968.

Cavaleri, L. and P.M. Rizzoli, Wind wave prediction in shallow water:- Theory and application, J. Geophys. Res., 86, 10,961$10,973,1981$.

Cardone, V.J., W.J. Pierson and E.G. Ward, Hindcasting the directional spectra of hurricane-generated waves, J. Pet. Technol, 261, 91-127, 1975 .

Chen, Y.H. and H. Wang, Numerical model for nonstationary shallow water wave spectral transformations, J. Geophys. Res., $88,9851-9863,1983$.

Collins, J.I., Prediction of shallow-water spectra, J. Geophys. Res., 77, 2693-2707, 1972.

Divoky, D., B. Le Méhauté and A. Lin, Breaking waves on gentle slopes, J. Geophys. Res., 75, 1681-1692, 1970.

Elliott, J.A., Microscale pressure fluctuations near waves being generated by wind, J. Fluid Mech., 54, 427-448, 1972.

Ewing, J.A., A numerical wave prediction method for the North Atlantic Ocean, Dtsch. Hydrog. Z., 24, 241-261, 1971.

Gelci, R., J. Cazalé and J. Vassal, J., Utilisation des diagrammes de propagation a la provision energitique de la houle, Bull. Inf. Comitz Cen. Oceanogr. Etudes Côtes, 8, 169-187, 1956.

Golding, B.W., A wave prediction system for real-time sea state forecasting, Q. J. R. Meteorol. Soc., 109, 393-416, 1983.

Gonzalez, F.I., T.W. Thompson, W.E. Brown Jr. and D.E. Weiseman, Seasat wind and wave observations of northeast Pacific hurricane Iva, August 13, 1987, J. Geophys. Res., 87, 3431-3438, 1982.

Greenwood, J.A., V.J. Cardone and L.M. Lawson, Intercomparison test version of the SAIL wave model, in The Sea Wave Modeling Project, pp. 221-233, SWAMP Group, Plenum, New York, 1985.

Günter, H., G.J. Komen and W. Rosenthal, A semi-operational comparison of two parametric wave prediction models, Dtsch Hydrogr. Z., s7, 90-106, 1984.

Hasselmann, D., M. Dunckel and J. Bösenberg, Measurements of atmospheric pressure over surface gravity waves during KonTur, in The Ocean Surface, edited by Y. Toba and H. Mitsuyasu, pp. 95-97, D.Reidel, Hingham, Mass., 1985.

Hasselmann, K., Grundgleichugen der Seegangevorhersage, Schiffstechnik, 7, 191-195, 1960.

Hasselmann, K., On the nonlinear energy transfer in a gravity wave spectrum, part 1, General theory, J. Fluid Mech., 12, 481$500,1962$.

Hasselmann, K. and J.I. Collins, Spatial dissipation of flnitedepth gravity waves due to turbulent bottom friction, $J$. Mor. Res., 26, 1-12, 1968.

Hasselmann, K., et al., Measurements of wind wave growth and swell decay during the Joint North Sea Wave Project, Reihe $A$, 12, 95 pp., D. Hydrogr. Inst., Hamburg, 1973.

Hasselmann, K., et al., A parametric wave prediction model, $J$. Phys. Oceanogr., 6, 200-228, 1976.

Hasselmann, S. and K. Hasselmann, A oymmetrical method of computing the nonlinear transfer in a gravity wave spectrum, Hamb. Geophys. Einzelschrift., Reihe A, 52, 157 pp.,Hamburg, 1981 .

Hasselmann, S., K. Hasselmann, J.H. Allender and T.P. Barnett, Computations and parameterizations of the nonlinear energy transfer in a gravity-wave spectrum, part II, Parameterizations of the nonlinear energy transfer for applications in wave models, J. Phys. Oceanogr., 15, 1378-1391, 1985.

Holt, B. and F.I. Gonzalez, SIR-B observations of dominant ocean waves near hurricane Josephine, J. Geophys. Res., 91, 8595-8598, 1986.

Hsiao, S.V. and O.H. Shemdin, Measurements of wind velocity and pressure with a wave follower during MARSEN, J. Geophys. Res., 88, 9841-9849, 1983.

Inoue, $T$., On the growth of the spectrum of a wind generated sea according to a modified Miles-Phillips mechanism and its application to wave forcasting, Ph.D. dissertation, New York University, New York, 1967.

Isozaki, I. and T. Uji, Numerical prediction of ocean wind waves, Pap. Meteonol. Geophys., 24, 207-231, 1973.

King, D.B. and O.H. Shemdin, Radar observations of hurricane wave directions, Proc. Conf. Coastal Eng., 16th, 109-226, 1978.

Kitaigorodskii, S.A., V.P. Krasitskii, and M.M. Zaslavskii, On Phillips' theory of equilibrium range in the spectra of windgenerated gravity waves, J. Phys. Oceanogr., 5, 410-420, 1975.

Komen, G.J., Recent results with a third-generation ocean wave model, APL Tech. Dig., 8, 37-41, 1987.

Komen, G.J., S. Hasselmann and K. Hasselmann, On the existence of a fully developed wind-sea spectrum, J. Phys. Oceanogr. 11, 1271-1285, 1984.

McLeish, W. and D.B. Ross, Imaging radar observations of directional properties of ocean waves, J. Geophys. Res., 88, 4407-4419, 1983.

Mitsuyasu, H., F. Tasai, T. Suhara, S. Mizuno, M. Ohkusu, T. Honda and $K$. Rikiishi, Observations of the power spectrum of ocean waves using a cloverleaf buoy, J. Phys. Oceanogr., 10, 286-296, 1980.

Munk, W.H. and R.S. Arthur, Wave intensity along a refracted ray, Gravity Waves, U.S. Nat. Bur. Stand, Cin. 521, 95-108, 1952.

Phillips, O.M., On the generation of waves by turbulent wind, $J$. Fluid Mech., $2,417-445,1957$.

Phillips, O.M., The Dynamice of the Upper Ocean, 2nd ed., Cambridge University Press, New York, 1977.

Phillips, O.M., Spectral and statistical properties of the equilibrium range in wind-generated gravity waves", J. Fluid Mech, $156,505-531,1985$.

Pierson, W.J., L.G. Tick and L. Baer, Computer-based procedures for predicting global wave forecasts and wind field analyses capable of using wave data obtained by spacecraft, paper presented at 6th Naval Hydrodynamics Symposium, Washington, 1966.

Plant, W.J., A relationship between wind stress and wave slope, J. Geophys. Res., 87, 1961-1967, 1982.

Snyder, R.L. and C.S. Cox, A field study of the wind generation of ocean waves, J. Mar. Res., 24, 141-178, 1966.

Snyder, R.L., et al., Array measurements of atmospheric pressure fluctuations above surface gravity waves, J. Fluid Mech., 102, 1$59,1981$.

Sobey, R.J., Wind-wave prediction, Annu. Rev. Fluid Mech., 16, 149-172, 1986.

Sobey, R.J. and I.R. Young, Hurricane wind waves-A discrete spectral model, J. Waterw. Port Coastal Ocean Eng., Am. Soc. Civ. Eng., 112(9), 370-389, 1986.

SWAMP Group, The Sea Wave Modeling Project (SWAMP): Principal Results and Conclusions, 256 pp., Plenum, New York, 1985.

Young, I.R., A general purpose spectral wave prediction model, Res. Rep. 16, Dep. Civ. Eng., Aust. Defence Force Acad., Canberra, 1987a.

Young, I.R., Validation of the spectral wave model ADFA1, Res. Rep. 17, Dep. Civ. Eng., Aust. Defence Force Acad., Canberra, $1987 b$.

Young, I.R. and R.J. Sobey, Measurements of the wind-wave energy flux in an opposing wind, J. Fluid Mech, 151, 427-442, 1985.

Young, I.R., S. Hasselmann and K. Hasselmann, Calculation of the nonlinear wave-wave interactions in cross seas, Hamb. Geophys. Einzelschrift., 74, 50 pp., 1985.

Young, I.R., S. Hasselmann and K. Hasselmann, Computations of the response of a wave spectrum to a sudden change in wind direction, J. Phys. Oceanogr., 17, 1317-1338, 1987.

I.R. Young, Department of Civil Engineering, Australian Defence Force Academy, Northcott Drive, Canberra, ACT, Australia, 2600.
(Received June 26, 1987; accepted November 24, 1987.) 